REPRESENTATION THEORY

An Electronic Journal of the American Mathematical Society

Volume 19, Pages 211-235 (October 28, 2015)

http://dx.doi.org/10.1090/ert/468

\title{
UNIPOTENT REPRESENTATIONS AS A CATEGORICAL CENTRE
}

\author{
G. LUSZTIG
}

\begin{abstract}
Let $G\left(F_{q}\right)$ be the group of rational points of a split connected reductive group $G$ over the finite field $F_{q}$. In this paper we show that the category of representations of $G\left(F_{q}\right)$ which are finite direct sums of unipotent representations in a fixed two-sided cell is equivalent to the centre of a certain monoidal category of sheaves on the flag manifold of $G \times G$. We also consider a version of this for nonsplit groups.
\end{abstract}

\section{INTRODUCTION}

0.1. Let $\mathbf{k}$ be an algebraic closure of the finite field $\mathbf{F}_{p}$ with $p$ elements. For any power $q$ of $p$ let $\mathbf{F}_{q}$ be the subfield of $\mathbf{k}$ with $q$ elements. Let $G$ be a reductive connected group over $\mathbf{k}$, assumed to be adjoint. Let $\mathcal{B}$ be the variety of Borel subgroups of $G$.

Let $W$ be the Weyl group of $G$ and let $\mathbf{c}$ be a two-sided cell of $W$. Let $s \in$ $\mathbf{Z}_{>0}$ and let $F: G \rightarrow G$ be the Frobenius map for an $\mathbf{F}_{p^{s}}$-rational structure on $G$. Let $G\left(\mathbf{F}_{p^{s}}\right)=G^{F}=\{g \in G ; F(g)=g\}$ be a finite group. Let $\operatorname{Rep}\left(G^{F}\right)$ (resp. $\left.\operatorname{Rep}^{\mathbf{c}}\left(G^{F}\right)\right)$ be the category of representations of $G^{F}$ over $\overline{\mathbf{Q}}_{l}$ which are finite direct sums of unipotent representations in the sense of [DL] (resp., of unipotent representations whose associated two-sided cell (see 1.3) is c); here $l$ is a fixed prime number invertible in $\mathbf{k}$.

In the rest of this subsection we assume for simplicity that the $\mathbf{F}_{p^{s}}$-rational structure on $G$ is split. The simple objects of $\operatorname{Rep}^{\mathbf{c}}\left(G^{F}\right)$ were classified in [L1]. The classification turns out to be the same as that [L4] of unipotent character sheaves on $G$ whose associated two-sided cell is c. The fact that

(a) these two classification problems have the same solution has not until now been adequately explained.

One of the guiding ideas of this paper (already formulated in [L12]) is that the theory of unipotent character sheaves on $G$ should be regarded as the limit for $s \mapsto 0$ of the theory of unipotent representations of the finite group $G\left(\mathbf{F}_{p^{s}}\right)$; equivalently, the theory of unipotent representations of $G\left(\mathbf{F}_{p^{s}}\right)$ should be regarded as a $q$-analogue or quantum version of the theory of character sheaves on $G$.

In L13] we have shown that the category of perverse sheaves on $G$ which are direct sums of unipotent character sheaves with associated two-sided cell $\mathbf{c}$ is naturally equivalent to the centre of a certain monoidal category $\mathcal{C}^{\mathbf{c}} \mathcal{B}^{2}$ of sheaves on $\mathcal{B}^{2}$ introduced in [L9] for which the induced ring structure on the Grothendieck group is the $J$-ring attached to c; see [L10, 18.3]. (The analogous statement for

Received by the editors December 5, 2014 and, in revised form, August 26, 2015.

2010 Mathematics Subject Classification. Primary 20G99.

Supported in part by National Science Foundation grant 1303060.

(C)2015 American Mathematical Society 
$D$-modules on a reductive group over $\mathbf{C}$ was proved earlier in a quite different way in $\left[\mathrm{BFO}\right.$.) In this paper we show that $\operatorname{Rep}^{\mathbf{c}}\left(G^{F}\right)$ is also naturally equivalent to the centre of $\mathcal{C}^{\mathbf{c}} \mathcal{B}^{2}$ (see Theorem 6.3).

This implies in particular that the set of simple objects of $\operatorname{Rep}^{\mathbf{c}}\left(G^{F}\right)$ (up to isomorphism) is naturally in bijection with the set of unipotent character sheaves (up to isomorphism) with associated two-sided cell c, which explains (a). Note that the bijection between these two sets is achieved by showing that both sets are in bijection with the set of simple objects of $\mathcal{C}^{\mathbf{c}} \mathcal{B}^{2}$ (up to isomorphism). (A less conceptual bijection between these two sets is given in [L11; we expect that it is the same as the bijection of this paper.) We deduce that the set of simple objects $\operatorname{Rep}_{\mathbf{c}}\left(G^{F}\right)$ is "independent" of the choice of $s$; in fact, as we show in 7.1, it is also independent of the characteristic of $\mathbf{k}$. It follows that to classify the unipotent representations of $G^{F}$ it is enough to classify the unipotent character sheaves on $G$ in sufficiently large characteristic; for the latter classification one can use the scheme of [L11] which uses the unipotent support of a character sheaf.

The methods of this paper are extensions of those in [L13]. We replace $\operatorname{Rep}^{\mathbf{c}} G^{F}$ by an equivalent category consisting of certain $G$-equivariant perverse sheaves on $G_{s}$, the set of all Frobenius maps $G \rightarrow G$ corresponding to split $F_{p^{s} \text {-rational struc- }}$ tures on $G$; we view $G_{s}$ as an algebraic variety in a natural way.

We construct functors $\underline{\chi}_{s}, \underline{\zeta}_{s}$ between this category and the category $\mathcal{C}^{\mathbf{c}} \mathcal{B}^{2}$ which are analogues of the truncated induction and truncated restriction $\underline{\chi}, \underline{\zeta}$ of [L13] and we show that most properties of $\chi, \zeta$ are preserved. We also define a truncated convolution product from our sheaves on $G_{s}$ and on $G_{s^{\prime}}$ to our sheaves on $G_{s+s^{\prime}}$ which is analogous to the truncated convolution of character sheaves in [L13] we also give a meaning for this even when $s, s^{\prime}$ are arbitrary integers. The main application of this truncated convolution product is in the case where $s^{\prime}=-s$, the result of the product being a direct sum of character sheaves on $G$; this is used in the proof of a weak form of an adjunction formula between $\underline{\chi}_{s}, \underline{\zeta}_{s}$ which is then used to prove the main result (Theorem 6.3).

0.2. In this paper we also prove extensions of the results in 0.1 to the case where $F: G \rightarrow G$ is the Frobenius map of a nonsplit $F_{p^{s}}$-rational structure. In this case the role of unipotent character sheaves on $G$ is taken by the unipotent character sheaves on a connected component of the group of automorphisms of $G$. Moreover, in this case the centre of $\mathcal{C}^{\mathbf{c}} \mathcal{B}^{2}$ is replaced by a slight generalization of the centre (the $\epsilon$-centre) which depends on the connected component above.

Many arguments in this paper are very similar to arguments in [L13] and are often replaced by references to the corresponding arguments in L13.

The analogues of our results in the case of nonunipotent character sheaves are considered in [L14].

0.3. Notation. We assume that we are given a split $\mathbf{F}_{p}$-rational structure on $G$ with Frobenius map $F_{0}: G \rightarrow G$. Let $\nu=\operatorname{dim} \mathcal{B}, \Delta=\operatorname{dim}(G), \rho=\operatorname{rk}(G)$. We shall view $W$ as an indexing set for the orbits of $G$ acting on $\mathcal{B}^{2}:=\mathcal{B} \times \mathcal{B}$ by simultaneous conjugation; let $\mathcal{O}_{w}$ be the orbit corresponding to $w \in W$ and let $\overline{\mathcal{O}}_{w}$ be the closure of $\mathcal{O}_{w}$ in $\mathcal{B}^{2}$. For $w \in W$ we set $|w|=\operatorname{dim} \mathcal{O}_{w}-\nu$ (the length of $w$ ). Let $w_{\max }$ be the unique element of $W$ such that $\left|w_{\max }\right|=\nu$.

As in [L1, 3.1], we say that an automorphism $\epsilon: W \rightarrow W$ is ordinary if it leaves stable the set $\{s \in W ;|s|=1\}$ and for any two elements $s \neq s^{\prime}$ in that set which are in the same orbit of $\epsilon$, the product $s s^{\prime}$ has order $\leq 3$. For example, if $W$ is of type $B_{2}, G_{2}$ or $F_{4}$ and $\epsilon: W \rightarrow W$ has order 2 and preserves $\{s \in W ;|s|=1\}$ (as 
in the case of Ree and Suzuki groups), then $\epsilon$ is not ordinary. Let $\mathfrak{A}$ be the group of ordinary automorphisms of $W$.

For $B \in \mathcal{B}$, let $U_{B}$ be the unipotent radical of $B$. Then $B / U_{B}$ is independent of $B$; it is "the" maximal torus $T$ of $G$. Let $\mathcal{X}$ be the group of characters of $T$.

Let Rep $W$ be the category of finite dimensional representations of $W$ over $\mathbf{Q}$; let $\operatorname{Irr} W$ be a set of representatives for the isomorphism classes of irreducible objects of $\operatorname{Rep} W$.

For an algebraic variety $X$ over $\mathbf{k}$ we denote by $\mathcal{D}(X)$ the bounded derived category of constructible $\overline{\mathbf{Q}}_{l}$-sheaves on $X$ ( $l$ is a fixed prime number invertible in k); let $\mathcal{M}(X)$ be the subcategory of $\mathcal{D}(X)$ consisting of perverse sheaves on $X$. If $X$ has a fixed $\mathbf{F}_{q}$-structure $X_{0}$, we denote by $\mathcal{D}_{m}(X)$ what in [BBD, 5.1.5] is denoted by $\mathcal{D}_{m}^{b}\left(X_{0}, \overline{\mathbf{Q}}_{l}\right)$. (When $X$ is $G, \mathcal{B}, \mathcal{O}_{w}$ or $\overline{\mathcal{O}}_{w}$, the subscript ${ }_{m}$ refers to the $\mathbf{F}_{p^{s_{0}}}$-structure defined by $F_{0}^{s_{0}}$ for a sufficiently large $s_{0}>0$.) Note that any object $K \in \mathcal{D}_{m}(X)$ can be viewed as an object of $\mathcal{D}(X)$ which will be denoted again by $K$. For $K \in \mathcal{D}(X)$ and $i \in \mathbf{Z}$ let $\mathcal{H}^{i} K$ be the $i$-th cohomology sheaf of $K, \mathcal{H}_{x}^{i} K$ its stalk at $x \in X$, and let $K^{i}$ be the $i$-th perverse cohomology sheaf of $K$. For $K \in \mathcal{D}(X)$ (or $\left.K \in \mathcal{D}_{m}(X)\right)$ and $n \in \mathbf{Z}$ we write $K[[n]]=K[n](n / 2)$ where $[n]$ is a shift and $(n / 2)$ is a Tate twist; we write $\mathfrak{D}(K)$ for the Verdier dual of $K$. Let $\mathcal{M}_{m}(X)$ be the subcategory of $\mathcal{D}_{m}(X)$ whose objects are in $\mathcal{M}(X)$. If $K \in \mathcal{M}_{m}(X)$ and $j \in \mathbf{Z}$ we denote by $\mathcal{W}^{j} K$ the subobject of $K$ which has weight $\leq j$ and is such that $K / \mathcal{W}^{j} K$ has weight $>j$; see $\mathrm{BBD}$, 5.3.5]. Let $g r_{j} K=\mathcal{W}^{j} K / \mathcal{W}^{j-1} K$ be the associated pure perverse sheaf of weight $j$. For $K \in \mathcal{D}_{m}(X)$ we shall often write $K^{\{i\}}$ instead of $g r_{i}\left(K^{i}\right)(i / 2)$; recall that $K^{\{i\}}$ is a semisimple perverse sheaf (being pure of weight zero).

If $K \in \mathcal{M}(X)$ and $A$ is a simple object of $\mathcal{M}(X)$ we denote by $(A: K)$ the multiplicity of $A$ in a Jordan-Hölder series of $K$.

Assume that $C \in \mathcal{D}_{m}(X)$ and that $\left\{C_{i} ; i \in I\right\}$ is a family of objects of $\mathcal{D}_{m}(X)$. We shall write $C \approx\left\{C_{i} ; i \in I\right\}$ if the following condition is satisfied: there exist distinct elements $i_{1}, i_{2}, \ldots, i_{s}$ in $I$, objects $C_{j}^{\prime} \in \mathcal{D}_{m}(X)(j=0,1, \ldots, s)$ and distinguished triangles $\left(C_{j-1}^{\prime}, C_{j}^{\prime}, C_{i_{j}}\right)$ for $j=1,2, \ldots, s$ such that $C_{0}^{\prime}=0, C_{s}^{\prime}=C$; moreover, $C_{i}=0$ unless $i=i_{j}$ for some $j \in[1, s]$.

If $X, X^{\prime}$ are algebraic varieties over $\mathbf{k}$, we say that a map of sets $f: X \rightarrow X^{\prime}$ is a quasi-morphism if for some $\mathbf{F}_{q}$-rational structure on $X$ and $X^{\prime}$ with Frobenius maps $F$ and $F^{\prime}$ and some integer $t \geq 0, f F^{t}: X \rightarrow X^{\prime}$ is a morphism equal to $F^{\prime t} f$. If, in addition, $f F=F^{\prime} f$, then we have well-defined functors $f_{!}: \mathcal{D}_{m}(X) \rightarrow \mathcal{D}_{m}\left(X^{\prime}\right), f^{*}: \mathcal{D}_{m}\left(X^{\prime}\right) \rightarrow \mathcal{D}_{m}(X)$ such that $f_{!}$is the composition of usual functors $\left(f F^{t}\right)_{!}\left(F^{t}\right)^{*}=\left(F^{\prime t}\right)^{*}\left(F^{\prime t} f\right)$ ! and $f^{*}$ is the composition of usual functors $\left(F^{t}\right) !\left(f F^{t}\right)^{*}=\left(F^{\prime t} f\right)^{*}\left(F^{\prime t}\right) !$. The usual properties of $f_{!}, f^{*}$ for morphisms continue to hold for quasi-morphisms.

We will denote by $\mathbf{p}$ the variety consisting of one point. For any variety $X$ let $\mathfrak{L}_{X}=\alpha_{!} \overline{\mathbf{Q}}_{l} \in \mathcal{D}_{m} X$ where $\alpha: X \times T \rightarrow X$ is the obvious projection. We sometimes write $\mathfrak{L}$ instead of $\mathfrak{L}_{X}$. For example, we have $\mathfrak{L}_{\mathbf{p}}=\alpha_{!} \overline{\mathbf{Q}}_{l}$ where $\alpha: T \rightarrow \mathbf{p}$ is the obvious map.

Let $v$ be an indeterminate. For any $\phi \in \mathbf{Q}\left[v, v^{-1}\right]$ and any $k \in \mathbf{Z}$ we write $(k ; \phi)$ for the coefficient of $v^{k}$ in $\phi$. Let $\mathcal{A}=\mathbf{Z}\left[v, v^{-1}\right]$. 


\section{CONTEnTs}

1. Truncated induction

2. Truncated restriction

3. Truncated convolution from $G_{\epsilon, s} \times G_{\epsilon^{\prime}, s^{\prime}}$ to $G_{\epsilon \epsilon^{\prime}, s+s^{\prime}}$

4. Analysis of the composition $\underline{\zeta}_{\epsilon, s} \underline{\chi}_{\epsilon, s}$

5. Adjunction formula (weak form)

6. Equivalence of $\mathcal{C}^{\mathbf{c}} G_{\epsilon, s}$ with the $\epsilon$-centre of $\mathcal{C}^{\mathbf{c}} \mathcal{B}^{2}$

7. Relation with Soergel bimodules

\section{TRUnCATED IndUCtion}

1.1. For $y \in W$ let $L_{y} \in \mathcal{D}_{m}\left(\mathcal{B}^{2}\right)$ be the constructible sheaf which is $\overline{\mathbf{Q}}_{l}$ (with the standard mixed structure of pure weight 0 ) on $\mathcal{O}_{y}$ and is 0 on $\mathcal{B}^{2}-\mathcal{O}_{y}$; let $L_{y}^{\sharp} \in \mathcal{D}_{m}\left(\mathcal{B}^{2}\right)$ be its extension to an intersection cohomology complex of $\overline{\mathcal{O}}_{y}$ (equal to 0 on $\left.\mathcal{B}^{2}-\overline{\mathcal{O}}_{y}\right)$. Let $\mathbf{L}_{y}=L_{y}^{\sharp}[[|y|+\nu]] \in \mathcal{D}_{m}\left(\mathcal{B}^{2}\right)$. This is a simple perverse sheaf which is pure of weight 0 .

Let $r \geq 1$. For $\mathbf{w}=\left(w_{1}, w_{2}, \ldots, w_{r}\right) \in W^{r}$ we set $|\mathbf{w}|=\left|w_{1}\right|+\cdots+\left|w_{r}\right|$. For any $i<i^{\prime}$ in $[1, r]$ let $p_{i, i^{\prime}}: \mathcal{B}^{r+1} \rightarrow \mathcal{B}^{2}$ be the projection to the $i, i^{\prime}$ factors. From the definitions we see that

$$
L_{\mathbf{w}}^{[1, r]}:=p_{01}^{*} L_{w_{1}}^{\sharp} \otimes p_{12}^{*} L_{w_{2}}^{\sharp} \otimes p_{r-1, r}^{*} L_{w_{r}}^{\sharp} \in \mathcal{D}_{m}\left(\mathcal{B}^{r+1}\right)
$$

is the intersection cohomology complex of the projective variety

$$
\mathcal{O}_{\mathbf{w}}^{[1, r]}=\left\{\left(B_{0}, B_{1}, \ldots, B_{r}\right) \in \mathcal{B}^{r+1} ;\left(B_{i-1}, B_{i}\right) \in \overline{\mathcal{O}}_{w_{i}} \forall i \in[1, r]\right\}
$$

extended by 0 on $\mathcal{B}^{r+1}-\mathcal{O}_{\mathbf{w}}^{[1, r]}$ (it has the standard mixed structure of pure weight $0)$. For any $J \subset[1, r]$ we set

$$
\begin{aligned}
\mathcal{O}_{\mathbf{w}}^{J}=\{ & \left(B_{0}, B_{1}, \ldots, B_{r}\right) \in \mathcal{B}^{r+1} ;\left(B_{i-1}, B_{i}\right) \in \overline{\mathcal{O}}_{w_{i}} \forall i \in J,\left(B_{i-1}, B_{i}\right) \in \mathcal{O}_{w_{i}} \\
& \forall i \in[1, r]-J\} .
\end{aligned}
$$

Let $i_{J}: \mathcal{O}_{\mathbf{w}}^{J} \rightarrow \mathcal{O}_{\mathbf{w}}^{[1, r]}$ (resp. $i_{J}^{\prime}: \mathcal{O}_{\mathbf{w}}^{[1, r]}-\mathcal{O}_{\mathbf{w}}^{J} \rightarrow \mathcal{O}_{\mathbf{w}}^{[1, r]}$ ) be the obvious open (resp. closed) imbedding and let $L_{\mathbf{w}}^{J} \in \mathcal{D}_{m}\left(\mathcal{B}^{r+1}\right)\left(\operatorname{resp} . \quad \dot{L}_{\mathbf{w}}^{J} \in \mathcal{D}_{m}\left(\mathcal{B}^{r+1}\right)\right)$ be $i_{J}^{*} L_{\mathbf{w}}^{[1, r]}$ (resp. $\left.i_{J}^{\prime}{ }^{*} L_{\mathbf{w}}^{[1, r]}\right)$ extended by 0 on $\mathcal{B}^{r+1}-\mathcal{O}_{\mathbf{w}}^{J}\left(\operatorname{resp} . \mathcal{B}^{r+1}-\left(\mathcal{O}_{\mathbf{w}}^{[1, r]}-\mathcal{O}_{\mathbf{w}}^{J}\right)\right)$; we have a distinguished triangle

$$
\left(L_{\mathbf{w}}^{J}, L_{\mathbf{w}}^{[1, r]}, \dot{L}_{\mathbf{w}}^{J}\right)
$$

in $\mathcal{D}_{m}\left(\mathcal{B}^{r+1}\right)$.

For ${ }^{1} L,{ }^{2} L, \ldots,{ }^{r} L$ in $\mathcal{D}_{m}\left(\mathcal{B}^{2}\right)$ we set

$$
{ }^{1} L \bullet{ }^{2} L \bullet{ }^{r} L=p_{0 r !}\left(p_{01}^{*}{ }^{1} L \otimes p_{12}^{*}{ }^{2} L \otimes p_{r-1, r}^{*} L\right) \in \mathcal{D}_{m}\left(\mathcal{B}^{2}\right) .
$$

1.2. Let $\mathbf{H}$ be the free $\mathcal{A}$-module with basis $\left\{T_{w} ; w \in W\right\}$. It is well known that $\mathbf{H}$ has a unique structure of associative $\mathcal{A}$-algebra with $1=T_{1}$ (Hecke algebra) such that $T_{w} T_{w^{\prime}}=T_{w w^{\prime}}$ if $w, w^{\prime} \in W,\left|w w^{\prime}\right|=|w|+\left|w^{\prime}\right|$ and $T_{s}^{2}=1+\left(v-v^{-1}\right) T_{s}$ if $s \in W,|s|=1$. Let $\left\{c_{w} ; w \in W\right\}$ be the "new" basis of $\mathbf{H}$ defined as in [L10, 5.2] with $L(w)=|w|$. For example, if $w=s \in W,|s|=1$. we have $c_{s}=T_{s}+v^{-1}$.

For $x, y \in W$, the relations $x \preceq y, x \sim y, x \sim_{L} y$ on $W$ are defined as in L13. 1.3]. If $\mathbf{c}$ is a two-sided cell of $W$ and $w \in W$, the relations $w \preceq \mathbf{c}, \mathbf{c} \preceq w$, $w \prec \mathbf{c}, \mathbf{c} \prec w$ are defined as in [L13, 1.3]. If $\mathbf{c}, \mathbf{c}^{\prime}$ are two-sided cells of $W$, the 
relations $\mathbf{c} \preceq \mathbf{c}^{\prime}, \mathbf{c} \prec \mathbf{c}^{\prime}$ are defined as in [L13, 1.3]. Let $\mathbf{a}: W \rightarrow \mathbf{N}$ be the afunction in [L10, 13.6]. If $\mathbf{c}$ is a two-sided cell of $W$, then for all $w \in \mathbf{c}$ we have $\mathbf{a}(w)=\mathbf{a}(\mathbf{c})$ where $\mathbf{a}(\mathbf{c})$ is a constant.

Let $\mathbf{J}$ be the free $\mathbf{Z}$-module with basis $\left\{t_{z} ; z \in W\right\}$ with the structure of associative ring (with 1) as in [L13, 1.3]. For a two-sided cell $\mathbf{c}$ of $W$ let $\mathbf{J}^{\mathbf{c}}$ be the subgroup of $\mathbf{J}$ generated by $\left\{t_{z} ; z \in \mathbf{c}\right\}$; it is a subring of $\mathbf{J}$ with unit element $\sum_{d \in \mathbf{D}_{\mathbf{c}}} t_{d}$ where $\mathbf{D}_{\mathbf{c}}$ is the set of distinguished involutions of $\mathbf{c}$. We have $\mathbf{J}=\bigoplus_{\mathbf{c}} \mathbf{J}^{\mathbf{c}}$ as rings.

For $E \in \operatorname{Irr} W$ we define a simple $\mathbf{Q} \otimes \mathbf{J}$-module $E_{\infty}$ and a simple $\mathbf{Q}(v) \otimes_{\mathcal{A}} \mathbf{H}$ module $E(v)$ as in [L13, 1.3]; there is a unique two-sided cell $\mathbf{c}_{E}$ of $W$ such that $\mathbf{J}^{\mathbf{c}_{E}} E_{\infty} \neq 0$.

Let $\epsilon \in \mathfrak{A}$. Let $E \in \operatorname{Irr} W$. We say that $E \in \operatorname{Irr}_{\epsilon} W$ if $\operatorname{tr}(\epsilon(w), E)=\operatorname{tr}(w, E)$ for any $w \in W$. In this case there exists a linear transformation of finite order $e_{E}: E \rightarrow E$ such that $e_{E} w e_{E}^{-1}=\epsilon(w): E \rightarrow E$ for any $w \in W$; moreover $e_{E}$ is unique up to multiplication by -1 . See ([L1, 3.2]). For each $E \in \operatorname{Irr}_{\epsilon} W$ we choose $e_{E}$ as above. As a $\mathbf{Q}$-vector space we have $E_{\infty}=E, E(v)=\mathbf{Q}(v) \otimes_{\mathbf{Q}} E$; hence, if $E \in \operatorname{Irr}_{\epsilon} W, e_{E}: E \rightarrow E$ can be viewed as a Q-linear map (of finite order) $e_{E}: E_{\infty} \rightarrow E_{\infty}$ and as a $\mathbf{Q}(v)$-linear map (of finite order) $e_{E}: E(v) \rightarrow E(v)$. From the definitions we see that $e_{E} t_{w} e_{E}^{-1}=t_{\epsilon(w)}: E_{\infty} \rightarrow E_{\infty}$ and $e_{E} T_{w} e_{E}^{-1}=T_{\epsilon(w)}:$ $E(v) \rightarrow E(v)$ for any $w \in W$.

If $E \in \operatorname{Irr}_{\epsilon} W$, then $\epsilon\left(\mathbf{c}_{E}\right)=\mathbf{c}_{E}$. Let $\operatorname{Irr}_{\epsilon, \mathbf{c}} W=\left\{E \in \operatorname{Irr}_{\epsilon} W ; \mathbf{c}_{E}=\mathbf{c}\right\}$.

1.3. For any $\epsilon \in \mathfrak{A}, s \in \mathbf{Z}$ let $G_{\epsilon, s}$ be the set of bijections $F: G \rightarrow G$ such that

(i) if $s>0$, then $F$ is the Frobenius map for an $F_{p^{s}}$-rational structure on $G$;

(ii) if $s<0$, then $F^{-1}$ is the Frobenius map for an $F_{p^{-s}}$-rational structure on $G$;

(iii) if $s=0$, then $F$ is an automorphism of $G$;

moreover in each case (i)-(iii) we require that the following holds: for any $w \in W$ and any $\left(B, B^{\prime}\right) \in \mathcal{O}_{w}$ we have $\left(F(B), F\left(B^{\prime}\right)\right) \in \mathcal{O}_{\epsilon(w)}$.

(If $\epsilon=1, s=0$ we can identify $G$ and $G_{\epsilon, s}$ by $g \mapsto \operatorname{Ad}(g)$; recall that $G$ is assumed to be adjoint.) Now $G$ acts on $G_{\epsilon, s}$ by $g: F \mapsto \operatorname{Ad}(g) F \operatorname{Ad}\left(g^{-1}\right)$. If $s \neq 0$, this action is transitive and the stabilizer of a point $F \in G_{\epsilon, s}$ is the finite group $G^{F}=\{g \in G ; F(g)=g\}$. (The transitivity follows from the classification of $\mathbf{F}_{q}$-rational structures on $G$ where $q=p^{s}$ if $s>0$ and $q=p^{-s}$ if $s<0$.)

For any $s \in \mathbf{Z}$ and any $\tilde{F} \in G_{\epsilon, s}$, the maps $\ell: G \rightarrow G_{\epsilon, s}, g \mapsto \operatorname{Ad}(g) \tilde{F}$ and $\ell^{\prime}: G \rightarrow G_{\epsilon, s}, g \mapsto \tilde{F} \operatorname{Ad}(g)$ are bijections. (The fact that $\ell, \ell^{\prime}$ are injective is obvious. We show that $\ell, \ell^{\prime}$ are surjective. This is obvious when $s=0$. Assume now that $s \neq 0$. If $F \in G_{\epsilon, s}$, we can write, by the transitivity property above, $F=\operatorname{Ad}(h) \tilde{F} \operatorname{Ad}\left(h^{-1}\right)$ for some $h \in G$. Thus, $F=\operatorname{Ad}(g) \tilde{F}$ where $g=-\tilde{F}\left(h^{-1}\right) \in G$ and $F=\tilde{F} \operatorname{Ad}\left(g^{\prime}\right)$ where $g^{\prime}=\tilde{F}^{-1}(h) h^{-1} \in G$, proving the surjectivity of $\ell$ and $\ell^{\prime}$.)

We use $\ell$ (resp. $\ell^{\prime}$ ) to view $G_{\epsilon, s}$ with $s \geq 0$ (resp. $s \leq 0$ ) as an affine algebraic variety isomorphic to $G$; this algebraic variety structure on $G_{\epsilon, s}$ is independent of the choice of $\tilde{F}$. We have $\operatorname{dim} G_{\epsilon, s}=\Delta$. When $X=G_{\epsilon, s}$ then the subscript ${ }_{m}$ in

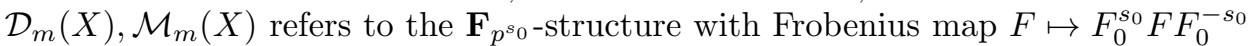
(with $F_{0}, s_{0}$ as in 0.3 ).

Note that $\bigsqcup_{\epsilon \in \mathfrak{A}, s \in \mathbf{Z}} G_{\epsilon, s}$ is a group under composition of maps: if $F \in G_{\epsilon, s}, F^{\prime} \in$ $G_{\epsilon^{\prime}, s^{\prime}}$, then $F F^{\prime} \in G_{\epsilon \epsilon^{\prime}, s+s^{\prime}}$. (It is enough to show that for some $F \in G_{\epsilon, s}, F^{\prime} \in$ $G_{\epsilon^{\prime}, s^{\prime}}$ we have $F F^{\prime} \in G_{\epsilon \epsilon^{\prime}, s+s^{\prime}}$. We take $F=\operatorname{Ad}(\gamma) F_{0}^{s}, F^{\prime}=\operatorname{Ad}\left(\gamma^{\prime}\right) F_{0}^{s^{\prime}}$ where $\gamma \in G_{\epsilon, 1}$ and $\gamma^{\prime} \in G_{\epsilon^{\prime}, 1}$ commute with $F_{0}$; then $F F^{\prime}=\operatorname{Ad}\left(\gamma \gamma^{\prime}\right) F_{0}^{s+s^{\prime}}$ and $\gamma \gamma^{\prime} \in$ $G_{\epsilon \epsilon^{\prime}, 1}$ commutes with $F_{0}$ hence $F F^{\prime} \in G_{\epsilon \epsilon^{\prime}, s+s^{\prime}}$.) Note that the composition $G_{\epsilon, s} \times$ 
$G_{\epsilon^{\prime}, s^{\prime}} \rightarrow G_{\epsilon \epsilon^{\prime}, s+s^{\prime}}$ is not in general a morphism of algebraic varieties but only a quasi-morphism (see 0.3), which is good enough for our purposes.

Until the end of Section 2 we fix $\epsilon \in \mathfrak{A}$.

Let $s \in \mathbf{Z}$. We consider the maps $\mathcal{B}^{2} \stackrel{f}{\longleftarrow} X_{\epsilon, s} \stackrel{\pi}{\longrightarrow} G_{\epsilon, s}$ where

$$
\begin{aligned}
& X_{\epsilon, s}=\left\{\left(B, B^{\prime}, F\right) \in \mathcal{B} \times \mathcal{B} \times G_{\epsilon, s} ; F(B)=B^{\prime}\right\}, \\
& f\left(B, B^{\prime}, F\right)=\left(B, B^{\prime}\right), \pi\left(B, B^{\prime}, F\right)=F .
\end{aligned}
$$

Note that $f, \pi$ are $G$-equivariant where $G$ acts on $G_{\epsilon, \sigma}$ by $g: F \mapsto \operatorname{Ad}(g) F \operatorname{Ad}\left(g^{-1}\right)$ and on $X_{\epsilon, s}$ by $g:\left(B, B^{\prime}, F\right) \mapsto\left(g B g^{-1}, g B^{\prime} g^{-1}, \operatorname{Ad}(g) F \operatorname{Ad}\left(g^{-1}\right)\right.$.

Now $L \mapsto \chi_{\epsilon, s}(L)=\pi_{!} f^{*} L$ defines a functor $\mathcal{D}_{m}\left(\mathcal{B}^{2}\right) \rightarrow \mathcal{D}_{m}\left(G_{\epsilon, s}\right)$. (When $\epsilon=1, s=0, c_{\epsilon, s}$ coincides with the functor $\chi$ defined in [L13, 1.5]). For $i \in \mathbf{Z}, L \in$ $\mathcal{D}_{m}\left(\mathcal{B}^{2}\right)$ we write $\chi_{\epsilon, s}^{i}(L)$ instead of $\left(\chi_{\epsilon, s}(L)\right)^{i}$, the $i$-th perverse cohomology sheaf of $\chi_{\epsilon, s}(L)$. For any $z \in W$ we set $R_{\epsilon, s, z}=\chi_{\epsilon, s}\left(L_{z}^{\sharp}\right) \in \mathcal{D}_{m}\left(G_{\epsilon, s}\right)$. (When $\epsilon=1, s=0$ this is the same as $R_{z}$ in [L13, 1.5].)

Let $b: G_{\epsilon^{-1},-s} \stackrel{\sim}{\longrightarrow} G_{\epsilon, s}$ be the isomorphism $F \mapsto F^{-1}$ and let $b^{\prime}: \mathcal{B}^{2} \stackrel{\sim}{\longrightarrow} \mathcal{B}^{2}$ be the isomorphism $\left(B, B^{\prime}\right) \mapsto\left(B^{\prime}, B\right)$. Using the definitions and base change, we see that for $L \in \mathcal{D}_{m}\left(\mathcal{B}^{2}\right)$ we have $\chi_{\epsilon, s}\left(b_{!}^{\prime} L\right)=b_{!} \chi_{\epsilon^{-1},-s}(L)$.

Let $C S\left(G_{\epsilon, s}\right)$ be a set of representatives for the isomorphism classes of simple perverse sheaves $A \in \mathcal{M}\left(G_{\epsilon, s}\right)$ such that $\left(A: R_{\epsilon, s, z}^{j}\right) \neq 0$ for some $z \in W, j \in \mathbf{Z}$. (When $\epsilon=1, s=0$ this agrees with the definition of $C S(G)$ in [L13, 1.5].) Now let $A \in C S\left(G_{\epsilon, s}\right)$. We associate to $A$ a two-sided cell $\mathbf{c}_{A}$ as follows.

Assume first that $s \neq 0$. Since $A$ is $G$-equivariant and the conjugation action of $G$ on $G_{\epsilon, s}$ is transitive, for any $F \in G_{\epsilon, s}$ we have $\left.A\right|_{\{F\}}=r_{A, F}[\Delta]$ where $r_{A, F}$ is an irreducible $G^{F}$-module. From the definitions, for any $z \in W$ and any $F \in G_{\epsilon, s}$ we have

$$
\left(A: R_{s, z}^{j}\right)=\left(r_{A, F}: I H^{j-\Delta}\left\{\left(B ;(B, F B) \in \overline{\mathcal{O}}_{z}\right\}\right)_{G^{F}}\right.
$$

where the right-hand side is the multiplicity of $r_{A, F}$ in the $G^{F}$-module

$$
I H^{j-\Delta}\left\{\left(B ;(B, F B) \in \overline{\mathcal{O}}_{z}\right\} ;\right.
$$

here $I H$ denotes intersection cohomology with coefficients in $\overline{\mathbf{Q}}_{l}$. In particular, $r_{A, F}$ is a unipotent representation of $G^{F}$. By [L1, 3.8], for any $A \in C S\left(G_{\epsilon, s}\right)$, any $F \in G_{\epsilon, s}$, any $z \in W$ and any $j \in \mathbf{Z}$ we have

$$
\begin{aligned}
& \left(r_{A, F}: I H^{j-\Delta}\left\{\left(B ;(B, F B) \in \overline{\mathcal{O}}_{z}\right\}\right)_{G^{F}}\right. \\
& =\left(j-\Delta-|z| ;(-1)^{j-\Delta} \sum_{E \in \operatorname{Irr}_{\epsilon} W} c_{A, E, e_{E}} \operatorname{tr}\left(e_{E} c_{z}, E(v)\right)\right)
\end{aligned}
$$

or, equivalently,

$$
\left(A: R_{\epsilon, s, z}^{j}\right)=\left(j-\Delta-|z| ;(-1)^{j-\Delta} \sum_{E \in \operatorname{Irr}_{\epsilon} W} c_{A, E, e_{E}} \operatorname{tr}\left(e_{E} c_{z}, E(v)\right)\right)
$$

where $c_{A, E, e_{E}}$ are uniquely defined rational numbers; now (a) also holds when $s=0$, see [L13, 1.5(a)] when $\epsilon=1$ and [L6, 34.19, 35.22], [L8, 44.7(e)] for general $\epsilon$. Moreover, if $s \neq 0$ then, by [L1, 6.17], given $A$ as above, there is a unique two-sided cell $\mathbf{c}_{A}$ of $W$ such that $\epsilon\left(\mathbf{c}_{A}\right)=\mathbf{c}_{A}$ and $c_{A, E, e_{E}}=0$ whenever $E \in \operatorname{Irr}_{\epsilon} W$ satisfies $\mathbf{c}_{E} \neq \mathbf{c}_{A}$. The same holds when $s=0$; see [L13, 1.5] when $\epsilon=1$ and [L7, §41] for general $\epsilon$.

When $s \neq 0, \mathbf{c}_{A}$ differs from the two-sided cell associated to $r_{A, F}$ in [L1, 4.23] by multiplication on the left or right by $w_{\max }$. (For example, if $G=P G L_{2}(\mathbf{k})$, 
then there are two objects $A_{0} \neq A_{1}$ in $C S\left(G_{\epsilon, s}\right)$ where $A_{0}$ corresponds to the unit representation and $A_{1}$ to the Steinberg representation. We have $\mathbf{c}_{A_{0}}=\left\{w_{\max }\right\}$, $\mathbf{c}_{A_{1}}=\{1\}$.) Similarly, when $s=0, \mathbf{c}_{A}$ differs from the two-sided cell associated to $A$ in [L7, $\S 41]$ by multiplication on the left or right by $w_{\max }$.

As in [L13, 1.5(b)], for $s \in \mathbf{Z}$ we have

(b) $\left(A: R_{\epsilon, s, z}^{j}\right) \neq 0$ for some $z \in \mathbf{c}_{A}, j \in \mathbf{Z}$ and conversely, if $\left(A: R_{\epsilon, s, z}^{j}\right) \neq 0$ for $z \in W, j \in \mathbf{Z}$, then $\mathbf{c}_{A} \preceq z$.

For $s \in \mathbf{Z}, A \in C S\left(G_{\epsilon, s}\right)$ let $a_{A}$ be the value of the a-function on $\mathbf{c}_{A}$. If $z \in W, E \in$ $\operatorname{Irr}_{\epsilon} W$ satisfy $\operatorname{tr}\left(e_{E} c_{z}, E(v)\right) \neq 0$, then $\mathbf{c}_{E} \preceq z$; if in addition we have $z \in \mathbf{c}_{E}$, then

$$
\operatorname{tr}\left(e_{E} c_{z}, E(v)\right)=\gamma_{z, E, e_{E}} v^{a_{E}}+\text { lower powers of } v
$$

where $\gamma_{z, E, e_{E}} \in \mathbf{Z}$ and $a_{E}$ is the value of the a-function on $\mathbf{c}_{E}$. Hence from (a) we see that

(c) $\left(A: R_{\epsilon, s, z}^{j}\right)=0$ unless $\mathbf{c}_{A} \preceq z$ and, if $z \in \mathbf{c}_{A}$, then

$$
\begin{aligned}
& \left(A: R_{\epsilon, s, z}^{j}\right) \\
& =(-1)^{j+\Delta}\left(j-\Delta-|z| ;\left(\sum_{E \in \operatorname{Irr}_{\epsilon} W ; \mathbf{c}_{E}=\mathbf{c}_{A}} c_{A, E, e_{E}} \gamma_{z, E, e_{E}}\right) v^{a_{A}}+\text { lower powers of } v\right)
\end{aligned}
$$

which is 0 unless $j-\Delta-|z| \leq a_{A}$.

In the remainder of this section we fix a two-sided cell $\mathbf{c}$ of $W$ such that $\epsilon(\mathbf{c})=\mathbf{c}$; we set $a=\mathbf{a}(\mathbf{c})$.

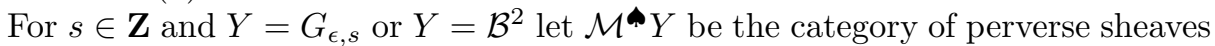
on $Y$ whose composition factors are all of the form $A \in C S\left(G_{\epsilon, s}\right)$, when $Y=G_{\epsilon, s}$, or of the form $\mathbf{L}_{z}$ with $z \in W$, when $Y=\mathcal{B}^{2}$. Let $\mathcal{M}^{\prec} Y$ (resp. $\mathcal{M}^{\prec} Y$ ) be the category of perverse sheaves on $Y$ whose composition factors are all of the form $A \in C S\left(G_{\epsilon, s}\right)$ with $\mathbf{c}_{A} \preceq \mathbf{c}$ (resp. $\mathbf{c}_{A} \prec \mathbf{c}$ ), when $Y=G_{\epsilon, s}$, or of the form $\mathbf{L}_{z}$ with $z \preceq$ c (resp. $z \prec$ c) when $Y=\mathcal{B}^{2}$. Let $\mathcal{D}^{\wedge} Y$ (resp. $\mathcal{D}^{\preceq} Y$ or $\mathcal{D}^{\prec} Y$ ) be the category of all $K \in \mathcal{D}(Y)$ such that $K^{i} \in \mathcal{M}^{\uparrow} Y$ (resp. $K^{i} \in \mathcal{M}^{\prec} Y$ or $K^{i} \in \mathcal{M}^{\prec} Y$ ) for all $i \in \mathbf{Z}$. Let $\mathcal{M}_{m}^{\bullet} Y$ (or $\mathcal{M}_{m}^{\prec} Y$, or $\mathcal{M}_{m}^{\prec} Y$ ) be the category of all $K \in \mathcal{M}_{m} Y$ which are also in $\mathcal{M}^{\mathfrak{c}} Y$ (or $\mathcal{M}^{\prec} Y$ or $\mathcal{M}^{\prec} Y$ ). Let $\mathcal{D}_{m}^{\wedge} Y$ (or $\mathcal{D}_{m}^{\prec} Y$, or $\mathcal{D}_{m}^{\prec} Y$ ) be the category of all $K \in \mathcal{D}_{m} Y$ which are also in $\mathcal{D}^{\wedge} Y$ (or $\mathcal{D}^{\preceq} Y$ or $\mathcal{D}^{\prec} Y$ ). From (c) we deduce:

(d) If $z \preceq \mathbf{c}$, then $R_{\epsilon, s, z}^{j} \in \mathcal{M} \preceq G_{\epsilon, s}$ for all $j \in \mathbf{Z}$. If $z \in \mathbf{c}$ and $j>a+\Delta+|z|$, then $R_{\epsilon, s, z}^{j} \in \mathcal{M}^{\prec} G_{\epsilon, s}$. If $z \prec \mathbf{c}$, then $R_{\epsilon, s, z}^{j} \in \mathcal{M}^{\prec} G_{\epsilon, s}$ for all $j \in \mathbf{Z}$.

Lemma 1.4. Let $s \in \mathbf{Z}$. Let $r \geq 1, J \subset[1, r], J \neq \emptyset$ and $\mathbf{w}=\left(w_{1}, w_{2}, \ldots, w_{r}\right) \in$ $W^{r}$.

(a) Assume that $w_{i} \in \mathbf{c}$ for some $i \in[1, r]$. If $j \in \mathbf{Z}$ (resp. $j>\Delta+$ ra), then $\chi_{\epsilon, s}^{j}\left(p_{0 r !} L_{\mathbf{w}}^{[1, r]}[|\mathbf{w}|]\right)$ is in $\mathcal{M} G_{\epsilon, s}$ (resp. $\left.\mathcal{M}^{\prec} G_{\epsilon, s}\right)$.

(b) Assume that $w_{i} \in \mathbf{c}$ for some $i \in J$. If $j \in \mathbf{Z}$ (resp. $j \geq \Delta+$ ra), then $\chi_{\epsilon, s}^{j}\left(p_{0 r !} \dot{L}_{\mathbf{w}}^{J}[|\mathbf{w}|]\right)$ is in $\mathcal{M} G_{\epsilon, s}$ (resp. $\left.\mathcal{M}^{\prec} G_{\epsilon, s}\right)$.

(c) Assume that $w_{i} \in \mathbf{c}$ for some $i \in J$. If $j \geq \Delta+$ ra, then the cokernel of the map

$$
\chi_{\epsilon, s}^{j}\left(p_{0 r !} L_{\mathbf{w}}^{J}[|\mathbf{w}|]\right) \rightarrow \chi_{\epsilon, s}^{j}\left(p_{0 r !} L_{\mathbf{w}}^{[1, r]}[|\mathbf{w}|]\right)
$$

associated to 1.1(a) is in $\mathcal{M}^{\prec} G_{\epsilon, s}$.

(d) Assume that $w_{i} \in \mathbf{c}$ for some $i \in J$. If $j \in \mathbf{Z}$ (resp. $j>\Delta+$ ra), then $\chi_{\epsilon, s}^{j}\left(p_{0 r !} L_{\mathbf{w}}^{J}[|\mathbf{w}|]\right)$ is in $\mathcal{M} \preceq G_{\epsilon, s}$ (resp. $\left.\mathcal{M}^{\prec} G_{\epsilon, s}\right)$.

(e) Assume that $w_{i} \prec \mathbf{c}$ for some $i \in J$. If $j \in \mathbf{Z}$, then $\chi_{\epsilon, s}^{j}\left(p_{0 r !} L_{\mathbf{w}}^{[1, r]}[|\mathbf{w}|]\right) \in$ $\mathcal{M}^{\prec} G_{\epsilon, s}$ and $\chi_{\epsilon, s}^{j}\left(p_{0 r !} L_{\mathbf{w}}^{J}[|\mathbf{w}|]\right) \in \mathcal{M}^{\prec} G_{\epsilon, s}$. 
When $\epsilon=1, s=0$ this is just [L13, 1.6]; the proof in the general case is entirely similar (it uses 1.3(b), 1.3(c)).

1.5. Let $s \in \mathbf{Z}$. Let $C S_{\epsilon, s, \mathbf{c}}=\left\{A \in C S\left(G_{\epsilon, s}\right) ; \mathbf{c}_{A}=\mathbf{c}\right\}$. For any $z \in \mathbf{c}$ we set $n_{z}=a+\Delta+|z|$. Let $A \in C S_{\epsilon, s, \mathbf{c}}$ and let $z \in \mathbf{c}$. We have:

$$
\left(A: R_{\epsilon, s, z}^{n_{z}}\right)=(-1)^{a+|z|} \sum_{E \in \operatorname{Irr}_{\epsilon, \mathrm{c}} W} c_{A, E, e_{E}} \operatorname{tr}\left(e_{E} t_{z}, E_{\infty}\right) .
$$

When $\epsilon=1, s=0$ this is just [L13, 1.7(a)]. In the general case, from 1.3(a) we have

$$
\left(A: R_{\epsilon, s, z}^{n_{z}}\right)=(-1)^{a+|z|} \sum_{E \in \operatorname{Irr}_{\epsilon} W} c_{A, E, e_{E}}\left(a ; \operatorname{tr}\left(e_{E} c_{z}, E(v)\right)\right)
$$

and it remains to use that $\left(a ; \operatorname{tr}\left(e_{E} c_{z}, E(v)\right)\right)$ is equal to $\operatorname{tr}\left(e_{E} t_{z}, E_{\infty}\right)$ if $E \in \operatorname{Irr}_{\epsilon, \mathbf{c}} W$ and to 0 , otherwise. We have:

(b) For any $A \in C S_{\epsilon, s, \mathbf{c}}$ there exists $z \in \mathbf{c}$ such that $\left(A: R_{\epsilon, s, z}^{n_{z}}\right) \neq 0$.

The proof, based on (a), is the same as that in the case $\epsilon=1, s=0$ given in L13, 1.7(b)].

Let $\mathbf{c}^{0}=\left\{z \in \mathbf{c} ; z \sim_{L} \epsilon(z 1)\right\}$. If $z \in \mathbf{c}-\mathbf{c}^{0}$ and $E \in \operatorname{Irr}_{\epsilon, \mathbf{c}} W$, then $\operatorname{tr}\left(e_{E} t_{z}, E_{\infty}\right)=0$. (We can write $E_{\infty}=\bigoplus_{d \in \mathbf{D}_{\mathrm{c}}} t_{d} E_{\infty}$ and $e_{E} t_{z}: E_{\infty} \rightarrow E_{\infty}$ maps the summand $t_{d} E_{\infty}$ (where $z \sim_{L} d$ ) into $t_{\epsilon\left(d^{\prime}\right)} E_{\infty}$ (where $d^{\prime} \in \mathbf{D}_{\mathbf{c}}, d^{\prime} \sim_{L} z^{-1}$ ) and all other summands to 0 . If $\operatorname{tr}\left(e_{E} t_{z}, E_{\infty}\right) \neq 0$, we must have $t_{d} E_{\infty}=t_{\epsilon\left(d^{\prime}\right)} E_{\infty} \neq 0$ and $d=\epsilon\left(d^{\prime}\right)$ and $z \sim_{L} \epsilon\left(z^{-1}\right)$.) From this and (a) we deduce:

(c) If $z \in \mathbf{c}-\mathbf{c}^{0}$, then $R_{\epsilon, s, z}^{n_{z}}=0$.

1.6. Let $s \in \mathbf{Z}$. For $Y=G_{\epsilon, s}$ or $\mathcal{B}^{2}$ let $\mathcal{C} \backsim$ be the subcategory of $\mathcal{M}^{\star} Y$ consisting of semisimple objects; let $\mathcal{C}_{0} Y$ be the subcategory of $\mathcal{M}_{m} Y$ consisting of those $K \in \mathcal{M}_{m} Y$ such that $K$ is pure of weight 0 (hence $K \in \mathcal{M}(Y)$ is semisimple) and such that as an object of $\mathcal{M}(Y), K$ belongs to $\mathcal{C}^{\top} Y$. Let $\mathcal{C}^{\mathbf{c}} Y$ be the subcategory of $\mathcal{M}^{\star} Y$ consisting of objects which are direct sums of objects in $C S_{\epsilon, s, \mathbf{c}}$ (if $\left.Y=G_{\epsilon, s}\right)$ or of the form $\mathbf{L}_{z}$ with $z \in \mathbf{c}$ (if $Y=\mathcal{B}^{2}$ ). Let $\mathcal{C}_{0}^{\mathbf{c}} Y$ be the subcategory of $\mathcal{C}_{0}^{\boldsymbol{h}} Y$ consisting of those $K \in \mathcal{C}_{0}^{\boldsymbol{m}} Y$ such that as an object of $\mathcal{C}^{\boldsymbol{c}} Y, K$ belongs to $\mathcal{C}^{\mathbf{c}} Y$. For $K \in \mathcal{C}_{0}^{\star} Y$, let $\underline{K}$ be the largest subobject of $K$ such that, as an object of $\mathcal{C}^{\star} Y$, we have $\underline{K} \in \mathcal{C}^{\mathbf{c}} Y$.

For $L \in \mathcal{C}_{0}^{\wedge} \mathcal{B}^{2}$ we define ${ }^{\epsilon} L \in \mathcal{C}_{0}^{\oplus} \mathcal{B}^{2}$ as follows. We have canonically $L=$ $\bigoplus_{y \in W} V_{y} \otimes \mathbf{L}_{y}$ where $V_{y}$ are finite dimensional $\overline{\mathbf{Q}}_{l}$-vector spaces; we set ${ }^{\epsilon} L=$ $\bigoplus_{y \in W} V_{y} \otimes \mathbf{L}_{\epsilon^{-1}(y)}$. We show:

(a) Let $s \in \mathbf{N}$. Define $u: G_{\epsilon, s} \times \mathcal{B}^{2} \rightarrow G_{\epsilon, s} \times \mathcal{B}^{2}$ by

$$
\left(F,\left(B_{1}, B_{2}\right)\right) \mapsto\left(F, F\left(B_{1}\right), F\left(B_{2}\right)\right)
$$

and let $L \in \mathcal{C}_{0}^{\boldsymbol{*}} \mathcal{B}^{2}$. We have canonically $u^{*}\left(\overline{\mathbf{Q}}_{l} \otimes L\right)=\overline{\mathbf{Q}}_{l} \otimes{ }^{\epsilon} L$. (Note that for $F \in G_{\epsilon, s}$, the perverse sheaf $F^{*} L$ is isomorphic to ${ }^{\epsilon} L$.)

We can assume that $L=\mathbf{L}_{y}$ where $y \in W$; we must show that $u^{*}\left(\overline{\mathbf{Q}}_{l} \otimes \mathbf{L}_{y}\right)=$ $\overline{\mathbf{Q}}_{l} \otimes \mathbf{L}_{\epsilon^{-1}(y)}$ or that $u^{*}\left(\overline{\mathbf{Q}}_{l} \otimes L_{y}^{\sharp}\right)=\overline{\mathbf{Q}}_{l} \otimes L_{\epsilon^{-1}(y)}^{\sharp}$. Now $\overline{\mathbf{Q}}_{l} \otimes L_{y}^{\sharp}$ is the intersection cohomology complex of $G_{\epsilon, s} \times \overline{\mathcal{O}}_{y}$ with coefficients in $\overline{\mathbf{Q}}_{l}$ (extended by 0 on $G_{\epsilon, s} \times$ $\left.\left(\mathcal{B}^{2}-\overline{\mathcal{O}}_{y}\right)\right)$. Hence $u^{*}\left(\overline{\mathbf{Q}}_{l} \otimes L_{y}^{\sharp}\right)$ is the intersection cohomology complex of $u^{-1}\left(G_{\epsilon, s} \times\right.$ $\overline{\mathcal{O}}_{y}$ ) with coefficients in $\overline{\mathbf{Q}}_{l}$ (extended by 0 on $G_{\epsilon, s} \times u^{-1}\left(\mathcal{B}^{2}-\overline{\mathcal{O}}_{y}\right)$ ); that is, the intersection cohomology complex of $G_{\epsilon, s} \times \overline{\mathcal{O}}_{\epsilon^{-1}(y)}$ with coefficients in $\overline{\mathbf{Q}}_{l}$ (extended by 0 on $\left.G_{\epsilon, s} \times\left(\mathcal{B}^{2}-\overline{\mathcal{O}}_{\epsilon^{-1}(y)}\right)\right)$. This is $\overline{\mathbf{Q}}_{l} \otimes L_{\epsilon^{-1}(y)}^{\sharp}$, as required. 
Assume that $s \in \mathbf{Z}_{>0}$ and let $F \in G_{\epsilon, s}$. For any $A \in \mathcal{C}^{\top} G_{\epsilon, s}$ we have $\left.A\right|_{\{F\}}=$ $r_{A, F}[\Delta]$ where $r_{A, F} \in \operatorname{Rep}\left(G^{F}\right)$ (see 0.1). Moreover, from the definitions we see that

(b) $A \mapsto r_{A, F}$ is an equivalence of categories $\mathcal{C}^{\mathbf{c}} G_{\epsilon, s} \stackrel{\sim}{\longrightarrow} \operatorname{Rep}^{\mathbf{c}}\left(G^{F}\right)$ (see 0.1).

Proposition 1.7. Let $s \in \mathbf{Z}$.

(a) If $L \in \mathcal{D} \preceq \mathcal{B}^{2}$, then $\chi_{\epsilon, s}(L) \in \mathcal{D} \preceq G_{\epsilon, s}$. If $L \in \mathcal{D}^{\prec} \mathcal{B}^{2}$, then $\chi_{\epsilon, s}(L) \in \mathcal{D}^{\prec} G_{\epsilon, s}$.

(b) If $L \in \mathcal{M} \preceq \mathcal{B}^{2}$ and $j>a+\nu+\rho$, then $\chi_{\epsilon, s}^{j}(L) \in \mathcal{M}^{\prec} G_{\epsilon, s}$.

When $\epsilon=1, s=0$ this is just [L13, 1.9]; the proof in the general case is entirely similar (it uses 1.4(a),(e)).

1.8. Let $s \in \mathbf{Z}$. For $L \in \mathcal{C}_{0}^{\mathbf{c}} \mathcal{B}^{2}$ we set

$$
\underline{\chi}_{\epsilon, s}(L)=\underline{\chi_{\epsilon, s}^{a+\nu+\rho}(L)}((a+\nu+\rho) / 2)=\underline{\left(\chi_{\epsilon, s}(L)\right)^{\{a+\nu+\rho\}} \in \mathcal{C}_{0}^{\mathbf{c}} G_{\epsilon, s} .}
$$

This is the projection onto $\mathcal{C}_{0}^{\mathbf{c}} G_{\epsilon, s}$ of the pure (of weight 0 ) semisimple perverse sheaf $\left(\chi_{\epsilon, s}(L)\right)^{\{a+\nu+\rho\}}$.

The functor $\underline{\chi}_{\epsilon, s}: \mathcal{C}_{0}^{\mathbf{c}} \mathcal{B}^{2} \rightarrow \mathcal{C}_{0}^{\mathbf{c}} G_{\epsilon, s}$ is called truncated induction. For $z \in \mathbf{c}$ we have

$$
\underline{\chi}_{\epsilon, s}\left(\mathbf{L}_{z}\right)=\underline{R_{\epsilon, s, z}^{n_{z}}}\left(n_{z} / 2\right) .
$$

When $\epsilon=1, s=0$ this is just [L13, 1.10(a)]; the proof in the general case is entirely similar.

We shall denote by $\tau: \mathbf{J}^{\mathbf{c}} \rightarrow \mathbf{Z}$ the group homomorphism such that $\tau\left(t_{z}\right)=1$ if $z \in \mathbf{D}_{\mathbf{c}}$ and $\tau\left(t_{z}\right)=0$, otherwise. For $z, u \in \mathbf{c}$ we have:

$$
\operatorname{dim} \operatorname{Hom}_{\mathcal{C}^{\mathbf{c}} G_{\epsilon, s}}\left(\underline{\chi}_{\epsilon, s}\left(\mathbf{L}_{z}\right), \underline{\chi}_{\epsilon, s}\left(\mathbf{L}_{u}\right)\right)=\sum_{y \in \mathbf{c}} \tau\left(t_{y^{-1}} t_{z} t_{\epsilon(y)} t_{u^{-1}}\right) .
$$

When $\epsilon=1, s=0$ this is just [L13, 1.10(b)]. We now consider the general case.

Using (a) and the definitions we see that the left-hand side of (b) equals

$$
\sum_{A \in C S_{\epsilon, s, \mathbf{c}}}\left(A: R_{\epsilon, s, z}^{n_{z}}\right)\left(A: R_{\epsilon, s, u}^{n_{u}}\right),
$$

hence, using 1.5(a) it equals

$$
\sum_{E, E^{\prime} \in \operatorname{Irr}_{\epsilon, \mathrm{c}} W}(-1)^{|z|+|u|} \sum_{A \in C S_{\epsilon, s, \mathbf{c}}} c_{A, E, e_{E}} c_{A, E^{\prime}, e_{E^{\prime}}} \operatorname{tr}\left(e_{E} t_{z}, E_{\infty}\right) \operatorname{tr}\left(e_{E^{\prime}} t_{u}, E_{\infty}^{\prime}\right) .
$$

Replacing in the last sum $\sum_{A \in C S_{\epsilon, s, \mathbf{c}}} c_{A, E, e_{E}} c_{A, E^{\prime}, e_{E^{\prime}}}$ by 1 if $E=E^{\prime}$ and by 0 if $E \neq E^{\prime}$ (see [L1, 3.9] in the case $s \neq 0$ and [L3, 13.12], [L6, 35.18(g)] in the case $s=0)$ we obtain

$$
\sum_{E \in \operatorname{Irr}_{\epsilon, \mathrm{c}} W}(-1)^{|z|+|u|} \operatorname{tr}\left(e_{E} t_{z}, E_{\infty}\right) \operatorname{tr}\left(e_{E} t_{u}, E_{\infty}\right) .
$$

This is equal to $(-1)^{|z|+|u|}$ times the trace of the operator $\xi \mapsto t_{z} \epsilon(\xi) t_{u^{-1}}$ on $\mathbf{Q} \otimes \mathbf{J}^{\mathbf{c}}$ (see [L6, 34.14(a), 34.17]). The last trace is equal to the sum over $y \in \mathbf{c}$ of the coefficient of $t_{y}$ in $t_{z} t_{\epsilon(y)} t_{u^{-1}}$; this coefficient is equal to $\tau\left(t_{y^{-1}} t_{z} t_{\epsilon(y)} t_{u^{-1}}\right)$ since for $y, y^{\prime} \in \mathbf{c}, \tau\left(t_{y^{\prime}} t_{y}\right)$ is 1 if $y^{\prime}=y^{-1}$ and is 0 if $y^{\prime} \neq y^{-1}$ (see [L10, 20.1(b)]). Thus we have

$$
\operatorname{dim} \operatorname{Hom}_{\mathcal{C}^{\mathrm{c}} G_{\epsilon, s}}\left(\underline{\chi}_{\epsilon, s}\left(\mathbf{L}_{z}\right), \underline{\chi}_{\epsilon, s}\left(\mathbf{L}_{u}\right)\right)=(-1)^{|u|+|z|} \sum_{y \in \mathbf{c}} \tau\left(t_{y^{-1}} t_{z} t_{\epsilon(y)} t_{u^{-1}}\right) .
$$


Since $\operatorname{dim} \operatorname{Hom}_{\mathcal{C}^{\mathbf{c}} G_{\epsilon, s}}\left(\underline{\chi}_{\epsilon, s}\left(\mathbf{L}_{z}\right), \underline{\chi}_{\epsilon, s}\left(\mathbf{L}_{u}\right)\right) \in \mathbf{N}$ and $\sum_{y \in \mathbf{c}} \tau\left(t_{y^{-1}} t_{z} t_{\epsilon(y)} t_{u^{-1}}\right) \in \mathbf{N}$, it follows that (b) holds.

The following lemma will be used several times to transfer properties of usual functors and operations to their truncated analogue.

Lemma 1.9. Let $s \in \mathbf{N}$. Let $Y_{1}, Y_{2}$ be among $G_{\epsilon, s}, \mathcal{B}^{2}$ and let $\mathbf{X} \in \mathcal{D}_{\bar{m}}^{\prec} Y_{1}$. Let $c, c^{\prime}$ be integers and let $\Phi: \mathcal{D}_{\bar{m}}^{\prec} Y_{1} \rightarrow \mathcal{D}_{m}^{\prec} Y_{2}$ be a functor which takes distinguished triangles to distinguished triangles, commutes with shifts, maps $\mathcal{D}_{m}^{\prec} Y_{1}$ into $\mathcal{D}_{m}^{\prec} Y_{2}$ and maps complexes of weight $\leq i$ to complexes of weight $\leq i$ (for any $i$ ). Assume that (a) and (b) below hold:

$$
\left(\Phi\left(\mathbf{X}_{0}\right)\right)^{h} \in \mathcal{M}_{m}^{\prec} Y_{2} \text { for any } \mathbf{X}_{0} \in \mathcal{M}_{m}^{\prec} Y_{1} \text { and any } h>c ;
$$

$$
\mathbf{X} \text { has weight } \leq 0 \text { and } \mathbf{X}^{i} \in \mathcal{M}^{\prec} Y_{1} \text { for any } i>c^{\prime} \text {. }
$$

Then

$$
(\Phi(\mathbf{X}))^{j} \in \mathcal{M}^{\prec} Y_{2} \text { for any } j>c+c^{\prime},
$$

and we have canonically

$$
\underline{\left(\Phi\left(\underline{\mathbf{X}^{\left\{c^{\prime}\right\}}}\right)\right)^{\{c\}}}=\underline{(\Phi(\mathbf{X}))^{\left\{c+c^{\prime}\right\}}} .
$$

When $\epsilon=1, s=0$ this is just [L13, 1.12]; the proof in the general case is entirely similar.

1.10. Let $s \in \mathbf{Z}$. Let $L \in \mathcal{C}_{0}^{\mathbf{c}} \mathcal{B}^{2}$. We have $\mathfrak{D}(L) \in \mathcal{C}_{0}^{\mathbf{c}} \mathcal{B}^{2}$. Moreover, we have canonically:

$$
\underline{\chi}_{\epsilon, s}(\mathfrak{D}(L))=\mathfrak{D}\left(\underline{\chi}_{\epsilon, s}(L)\right) .
$$

When $\epsilon=1, s=0$ this is just [L13, 1.13]; the proof in the general case is entirely similar.

1.11. Let $L \in \mathcal{C}_{0}^{\uparrow} \mathcal{B}^{2}, L^{\prime} \in \mathcal{D}_{m}\left(\mathcal{B}^{2}\right)$. We have canonically

$$
\chi_{\epsilon, s}\left(L \bullet L^{\prime}\right)=\chi_{\epsilon, s}\left(L^{\prime} \bullet{ }^{\epsilon} L\right) .
$$

In the case where $\epsilon=1, s=0$ this appears in [L13, 1.11]; the proof in the general case is entirely similar.

\section{TRUnCATED RESTRICTION}

2.1. Recall that $\epsilon \in \mathfrak{A}$ is fixed. In this section we fix $s \in \mathbf{Z}$. Let $\pi, f$ be as in 1.3. Now $K \mapsto \zeta_{\epsilon, s}(K)=f_{!} \pi^{*} K$ defines a functor $\mathcal{D}_{m}\left(G_{\epsilon, s}\right) \rightarrow \mathcal{D}_{m}\left(\mathcal{B}^{2}\right)$. (When $\epsilon=1, s=0, \zeta_{\epsilon, s}$ is the same as $\zeta$ of [L13, 2.5].) For $i \in \mathbf{Z}, K \in \mathcal{D}_{m}\left(G_{\epsilon, s}\right)$ we write $\zeta_{\epsilon, s}^{i}(K)$ instead of $\left(\zeta_{\epsilon, s}(K)\right)^{i}$ (the $i$-th perverse cohomology sheaf of $\zeta_{\epsilon, s}(K)$ ).

Let $b: G_{\epsilon^{-1},-s} \stackrel{\sim}{\longrightarrow} G_{\epsilon, s}, b^{\prime}: \mathcal{B}^{2} \stackrel{\sim}{\longrightarrow} \mathcal{B}^{2}$ be as in 1.3. From the definitions we see that for $K \in \mathcal{D}_{m}\left(G_{\epsilon^{-1},-s}\right)$ we have

$$
\zeta_{\epsilon, s}\left(b_{!} K\right)=b_{!}^{\prime} \zeta_{\epsilon^{-1},-s}(K) .
$$

Proposition 2.2. For any $L \in \mathcal{D}_{m}\left(\mathcal{B}^{2}\right)$ we have

$$
\zeta_{\epsilon, s}\left(\chi_{\epsilon, s}(L)\right) \approx\left\{\bigoplus_{y \in W ;|y|=k} L_{y} \bullet L \bullet L_{\epsilon(y)^{-1}} \otimes \mathfrak{L}[[2 k-2 \nu]] ; k \in \mathbf{N}\right\},
$$




$$
\begin{aligned}
& \zeta_{\epsilon, s}\left(\chi_{\epsilon, s}(L)\right) \approx \\
& \left\{\bigoplus_{y \in W ;|y|=k} L_{y} \bullet L \bullet L_{\epsilon(y)^{-1}}[[2 k-2 \nu-2 \rho]]\right. \\
& \left.\quad \otimes \Lambda^{d} \mathcal{X}[[d]](d / 2) ; k \in \mathbf{N}, d \in[0, \rho]\right\},
\end{aligned}
$$

where $\mathfrak{L}, \mathcal{X}$ are as in 0.3 .

When $\epsilon=1, s=0$ this is proved in [L13, 2.6]. The proof in the general case will be quite similar to that in the case $\epsilon=1, s=0$. Let

$$
Y=\left\{\left(B_{1}, B_{2}, B_{3}, B_{4}, F\right) \in \mathcal{B} \times \mathcal{B} \times \mathcal{B} \times \mathcal{B} \times G_{s} ; F\left(B_{1}\right)=B_{4}, F\left(B_{2}\right)=B_{3}\right\} .
$$

For $i j=14$ or 23 we define $h_{i j}^{\prime}: Y \rightarrow X_{\epsilon, s}$ by $\left(B_{1}, B_{2}, B_{3}, B_{4}, F\right) \mapsto\left(B_{i}, B_{j}, F\right)$ and $h_{i j}: Y \rightarrow \mathcal{B}^{2}$ by $\left(B_{1}, B_{2}, B_{3}, B_{4}, F\right) \mapsto\left(B_{i}, B_{j}\right)$. We have $\pi^{*} \pi_{!}=h_{14 !}^{\prime} h_{23}^{\prime}{ }^{*}$; hence

$$
\zeta_{\epsilon, s}\left(\chi_{\epsilon, s}(L)\right)=f_{!} \pi^{*} \pi_{!} f^{*}(L)=f_{!} h_{14 !}^{\prime} h_{23}^{\prime}{ }^{*} f^{*}(L)=h_{14 !} h_{23}^{*} L .
$$

For $k \in \mathbf{N}$ let $Y^{k}=\bigcup_{y \in W ;|y|=k} Y_{y}$ where

$$
Y_{y}=\left\{\left(B_{1}, B_{2}, B_{3}, B_{4}, F\right) \in Y ;\left(B_{1}, B_{2}\right) \in \mathcal{O}_{y},\left(B_{3}, B_{4}\right) \in \mathcal{O}_{\epsilon(y)^{-1}}\right\}
$$

and let $Y^{\geq k}:=\bigcup_{k^{\prime} ; k^{\prime} \geq k} Y^{k^{\prime}}$, an open subset of $Y$; let $h_{i j}^{k}: Y^{k} \rightarrow \mathcal{B}^{2}, h_{i j}^{\geq k}: Y \leq k \rightarrow$ $\mathcal{B}^{2}$ be the restrictions of $h_{i j}$. For any $k \in \mathbf{N}$ we have a distinguished triangle

$$
\left.\left(h_{14 !}^{\geq k+1} h_{23}^{\geq k+1 *} L\right), h_{14 !}^{\geq k} h_{23}^{\geq k *} L, h_{14 !}^{k} h_{23}^{k *} L\right) .
$$

It follows that we have

$$
\zeta_{\epsilon, s}\left(\chi_{\epsilon, s}(L)\right) \approx\left\{h_{14 !}^{k} h_{23}^{k *} L ; k \in \mathbf{N}\right\} .
$$

For $k \in \mathbf{N}$ let $Z^{k}=\bigcup_{y \in W ;|y|=k} Z_{y}$ where

$$
Z_{y}=\left\{\left(B_{1}, B_{2}, B_{3}, B_{4}\right) \in \mathcal{B}^{4} ;\left(B_{1}, B_{2}\right) \in \mathcal{O}_{y},\left(B_{3}, B_{4}\right) \in \mathcal{O}_{\epsilon(y)^{-1}}\right\}
$$

for $i, j \in[1,4]$ we define $\tilde{h}_{i j}^{k}: Z^{k} \rightarrow \mathcal{B}^{2}$ and $\tilde{h}_{i j}^{y}: Z_{y} \rightarrow \mathcal{B}^{2}$ by $\left(B_{1}, B_{2}, B_{3}, B_{4}\right) \mapsto$ $\left(B_{i}, B_{j}\right)$. We have an obvious morphism $u: Y^{k} \rightarrow Z^{k}$. The fibre of $u$ at $\left(B_{1}, B_{2}, B_{3}\right.$, $\left.B_{4}\right) \in Z^{k}$ can be identified with the set of all $F \in G_{\epsilon, s}$ such that $F\left(B_{1}\right)=$ $B_{4}, F\left(B_{2}\right)=B_{3}$. Since $\left(B_{1}, B_{2}\right) \in \mathcal{O}_{y},\left(B_{3}, B_{4}\right) \in \mathcal{O}_{\epsilon(y)^{-1}}$ for some $y \in W$, we can find $\tilde{F} \in G_{\epsilon, s}$ such that $\tilde{F}\left(B_{1}\right)=B_{4}, \tilde{F}\left(B_{2}\right)=B_{3}$; hence the fibre above can be identified with

$$
\begin{aligned}
& \left\{g \in G ; \operatorname{Ad}(g) \tilde{F}\left(B_{1}\right)=B_{4}, \operatorname{Ad}(g) \tilde{F}\left(B_{2}\right)=B_{3}\right\} \\
& =\left\{g \in G ; \operatorname{Ad}(g)\left(B_{4}\right)=B_{4}, \operatorname{Ad}(g)\left(B_{3}\right)=B_{3}\right\}=B_{3} \cap B_{4}
\end{aligned}
$$

if $s \geq 0$ and with

$$
\begin{aligned}
& \left\{g \in G ; \tilde{F} \operatorname{Ad}(g)\left(B_{1}\right)=B_{4}, \tilde{F} \operatorname{Ad}(g)\left(B_{2}\right)=B_{3}\right\} \\
& \quad=\left\{g \in G ; \operatorname{Ad}(g)\left(B_{1}\right)=B_{1}, \operatorname{Ad}(g)\left(B_{2}\right)=B_{2}\right\}=B_{1} \cap B_{2}
\end{aligned}
$$

if $s<0$, which is a $\mathbf{k}^{\nu-k}$-bundle over $T$ via the obvious map $B_{3} \cap B_{4} \rightarrow B_{4} / U_{B_{4}}=T$ (if $s \geq 0$ ) or $B_{1} \cap B_{2} \rightarrow B_{1} / U_{B_{1}}=T$ (if $s<0$ ). We have a commutative diagram

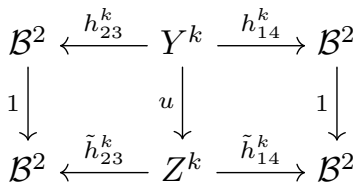


We have

$$
h_{14 !}^{k} h_{23}^{k *} L=\tilde{h}_{14 !}^{k} u ! u^{*} \tilde{h}_{23}^{k *} L=\tilde{h}_{14 !}^{k}\left(\tilde{h}_{23}^{k *} L \otimes u ! \overline{\mathbf{Q}}_{l}\right)=\left(\tilde{h}_{14 !}^{k} \tilde{h}_{23}^{k *} L\right) \otimes \mathfrak{L}[[-2 \nu+2 k]] .
$$

(The the last equality uses the description of the fibres of $u$ given above.) We deduce that

$$
\zeta_{\epsilon, s}\left(\chi_{\epsilon, s}(L)\right) \approx\left\{\left(\tilde{h}_{14 !}^{k} \tilde{h}_{23}^{k *} L\right) \otimes \mathfrak{L}[[-2 \nu+2 k]] ; k \in \mathbf{N}\right\} .
$$

Since $Z^{k}$ is the union of open and closed subvarieties $Z_{y},|y|=k$, we have

$$
\tilde{h}_{14 !}^{k} \tilde{h}_{23}^{k *} L=\bigoplus_{y \in W ;|y|=k} \tilde{h}_{14 !}^{y} \tilde{h}_{23}^{y *} L .
$$

From the definitions we have

$$
\tilde{h}_{14 !}^{y} \tilde{h}_{23}^{y *} L=L_{y} \bullet L \bullet L_{\epsilon(y)^{-1}} .
$$

This completes the proof of (a). Now (b) follows from (a) using

$$
\mathfrak{L}[[2 \rho]] \approx\left\{\overline{\mathbf{Q}}_{l} \otimes \Lambda^{d} \mathcal{X}[[d]](d / 2) ; d \in[0, \rho]\right\}
$$

which follows from the definitions.

In the remainder of this section we fix a two-sided cell $\mathbf{c}$ of $W$ such that $\epsilon(\mathbf{c})=\mathbf{c}$; we set $a=\mathbf{a}(\mathbf{c})$.

Proposition 2.3. Let $w \in W$ and let $j \in \mathbf{Z}$. We set $S=\zeta_{\epsilon, s}\left(R_{\epsilon, s, w}\right)[[2 \rho+2 \nu+$ $|w|]] \in \mathcal{D}_{m}\left(\mathcal{B}^{2}\right)$.

(a) If $w \preceq \mathbf{c}$, then $S^{j} \in \mathcal{M} \preceq \mathcal{B}^{2}$.

(b) If $w \in \mathbf{c}$ and $j>\nu+2 a$, then $S^{j} \in \mathcal{M}^{\prec} \mathcal{B}^{2}$.

(c) If $w \prec \mathbf{c}$, then $S^{j} \in \mathcal{M}^{\prec} \mathcal{B}^{2}$.

(d) $S^{j}$ is mixed of weight $\leq j$.

(e) If $j \neq \nu+2 a$ and $w \in \mathbf{c}$, then $g_{\nu+2 a} S^{j} \in \mathcal{M}^{\prec} \mathcal{B}^{2}$.

(f) If $k>\nu+2 a$ and $w \in \mathbf{c}$, then $g r_{k} S^{j} \in \mathcal{M}^{\prec} \mathcal{B}^{2}$.

When $\epsilon=1, s=0$ this is just [L13, 2.7]. The proof in the general case is entirely similar; it uses Proposition 2.2.

Proposition 2.4. (a) If $K \in \mathcal{D} \preceq G_{\epsilon, s}$, then $\zeta_{\epsilon, s}(K) \in \mathcal{D} \preceq \mathcal{B}^{2}$. If $K \in \mathcal{D}^{\prec} G_{\epsilon, s}$, then $\zeta_{\epsilon, s}(K) \in \mathcal{D}^{\prec} \mathcal{B}^{2}$.

(b) If $K \in \mathcal{M}^{\prec} G_{\epsilon, s}$ and $j>\rho+\nu+a$, then $\zeta_{\epsilon, s}^{j}(K) \in \mathcal{M}^{\prec} \mathcal{B}^{2}$.

When $\epsilon=1, s=0$ this is just [L13, 2.8]. The proof in the general case is similar. It is enough to prove (a) assuming in addition that $K=A \in C S_{\epsilon, s}(G)$. By 1.5(b) we can find $w \in \mathbf{c}$ such that $\left(A: R_{\epsilon, s, w}^{n_{w}}\right) \neq 0$. Then $A\left[-n_{w}\right]$ is a direct summand of $R_{\epsilon, s, w}$. Hence $\zeta_{\epsilon, s}(A)$ is a direct summand of $\zeta_{\epsilon, s}\left(R_{\epsilon, s, w}\right)[\Delta+a+|w|]$ and $\zeta_{\epsilon, s}^{j}(A)$ is a direct summand of

$$
\zeta_{\epsilon, s}^{j+\Delta+a+|w|}\left(R_{\epsilon, s, w}\right)=\zeta_{\epsilon, s}^{j-\rho+a}\left(R_{\epsilon, s, w}[2 \rho+2 \nu+|w|]\right) .
$$

Using Proposition 2.3 we deduce that (a) holds. A similar argument, based on 2.3, proves (b). 
2.5. For $K \in \mathcal{C}_{0}^{\mathbf{c}} G_{\epsilon, s}$ we set

$$
\underline{\zeta}_{\epsilon, s}(K)=\underline{\left(\zeta_{\epsilon, s}(K)\right)^{\{\rho+\nu+a\}} \in \mathcal{C}_{0}^{\mathbf{c}} \mathcal{B}^{2} .}
$$

We say that $\underline{\zeta}_{\epsilon, s}(K)$ is the truncated restriction of $K$.

Proposition 2.6. Let $K \in \mathcal{D}_{m}\left(G_{\epsilon, s}\right)$ and let $L \in \mathcal{C}_{0}^{\top} \mathcal{B}^{2}$. Then there is a canonical isomorphism ${ }^{\epsilon} L \bullet \zeta_{\epsilon, s}(K) \stackrel{\sim}{\longrightarrow} \zeta_{\epsilon, s}(K) \bullet L$.

When $\epsilon=1, s=0$ this follows from [L13, 2.10(a)]. We now consider the general case. Let $u: G_{\epsilon, s} \times \mathcal{B}^{2} \rightarrow G_{\epsilon, s} \times \mathcal{B}^{2}$ be as in 1.6(a). Using the base change theorem we have $\zeta_{\epsilon, s}(K) \bullet L=c_{!} d^{*}(K \otimes L)$ where $Z=\left\{\left(F,\left(B, B^{\prime \prime}, B^{\prime}\right)\right) \in\right.$ $\left.G_{\epsilon, s} \times \mathcal{B}^{3} ; F(B)=B^{\prime \prime}\right\}, d: Z \rightarrow G_{\epsilon, s} \times \mathcal{B}^{2}$ is $\left(F,\left(B, B^{\prime \prime}, B^{\prime}\right)\right) \mapsto\left(F,\left(B^{\prime \prime}, B^{\prime}\right)\right)$, $c: Z \rightarrow \mathcal{B}^{2}$ is $\left(F,\left(B, B^{\prime \prime}, B^{\prime}\right)\right) \mapsto\left(B, B^{\prime}\right)$. We have ${ }^{\epsilon} L \bullet \zeta_{\epsilon, s}(K)=c_{l}^{\prime} d^{\prime *}\left(K \otimes{ }^{\epsilon} L\right)$ where $Z^{\prime}=\left\{\left(F,\left(B, B^{\prime \prime}, B^{\prime}\right)\right) \in G_{\epsilon, s} \times \mathcal{B}^{3} ; F\left(B^{\prime \prime}\right)=B^{\prime}\right\}, d^{\prime}: Z^{\prime} \rightarrow G_{\epsilon, s} \times \mathcal{B}^{2}$ is $\left(F,\left(B, B^{\prime \prime}, B^{\prime}\right)\right) \mapsto\left(F,\left(B, B^{\prime \prime}\right)\right), c^{\prime}: Z^{\prime} \rightarrow \mathcal{B}^{2}$ is $\left(F,\left(B, B^{\prime \prime}, B^{\prime}\right)\right) \mapsto\left(B, B^{\prime}\right)$. Using 1.6(a) we have $K \nabla^{\epsilon} L=u^{*}(K \otimes L)$ hence it is enough to show that $c d^{*}(K \otimes L)=$ $c_{!}^{\prime} d^{\prime *} u^{*}(K \otimes L)$. We have $c_{!} d^{*}(K \otimes L)=c_{1 !} d_{1}^{*}(K \otimes L)=c_{1}^{\prime} d^{* *} u^{*}(K \otimes L)$ where $\dot{d}_{1}: G_{\epsilon, s} \times \mathcal{B}^{2} \rightarrow G_{\epsilon, s} \times \mathcal{B}^{2}$ is $\left(F,\left(B, B^{\prime}\right)\right) \mapsto\left(F,\left(F(B), B^{\prime}\right)\right), c_{1}: G_{\epsilon, s} \times \mathcal{B}^{2} \rightarrow \mathcal{B}^{2}$ is $\left(F,\left(B, B^{\prime}\right)\right) \mapsto\left(B, B^{\prime}\right)$. The proposition follows.

Proposition 2.7. (a) If $L \in \mathcal{M} \preceq \mathcal{B}^{2}$ and $j>2 a+2 \nu+2 \rho$, then $\left(\zeta_{\epsilon, s}\left(\chi_{\epsilon, s}(L)\right)\right)^{j} \in$ $\mathcal{M}^{\prec} \mathcal{B}^{2}$.

(b) If $L \in \mathcal{C}_{0}^{\mathbf{c}} \mathcal{B}^{2}$, we have canonically

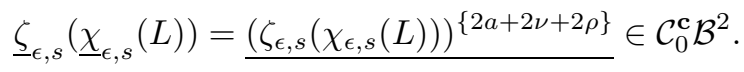

We apply Lemma 1.9 with $\Phi=\zeta_{\epsilon, s}: \mathcal{D}_{m}\left(G_{\epsilon, s}\right) \rightarrow \mathcal{D}_{m}\left(\mathcal{B}^{2}\right)$ and with $\mathbf{X}=\chi_{\epsilon, s}(L)$, $\left(c, c^{\prime}\right)=(a+\nu+\rho, a+\nu+\rho)$; see Propositions 2.4 and 1.7. The result follows.

2.8. For $L, L^{\prime} \in \mathcal{C}_{0}^{\mathbf{c}} \mathcal{B}^{2}$, we set (as in [L13, 3.2])

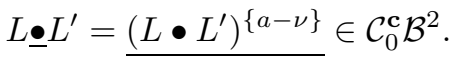

This defines an associative tensor product structure on $\mathcal{C}_{0}^{\mathbf{c}} \mathcal{B}^{2}$.

Proposition 2.9. Let $K \in \mathcal{C}_{0}^{\mathbf{c}} G_{\epsilon, s}, L \in \mathcal{C}_{0}^{\mathbf{c}} \mathcal{B}^{2}$. There is a canonical isomorphism

$$
{ }^{\epsilon} L \underline{\bullet}_{\epsilon, s}(K) \stackrel{\sim}{\longrightarrow} \underline{\zeta}_{\epsilon, s}(K) \underline{\bullet} L .
$$

Applying Lemma 1.9 with $\Phi: \mathcal{D}_{\bar{m}}^{\prec} \mathcal{B}^{2} \rightarrow \mathcal{D}_{\bar{m}}^{\prec} \mathcal{B}^{2}, L^{\prime} \mapsto L^{\prime} \bullet L, \mathbf{X}=\zeta_{\epsilon, s}(K)$, $\left(c, c^{\prime}\right)=(a-\nu, a+\rho+\nu)($ see [L13, 3.1] and 2.4), we deduce that we have canonically

$$
\underline{\left(\left(\zeta_{\epsilon, s}(K)\right)^{\{a+\rho+\nu\}} \bullet L\right)^{\{a-\nu\}}}=\underline{\left(\zeta_{\epsilon, s}(K) \bullet L\right)^{\{2 a+\rho\}}} .
$$

Using Lemma 1.9 with $\Phi: \mathcal{D}_{\bar{m}}^{\prec} \mathcal{B}^{2} \rightarrow \mathcal{D}_{\bar{m}}^{\prec} \mathcal{B}^{2}, L^{\prime} \mapsto{ }^{\epsilon} L \bullet L^{\prime}, \mathbf{X}=\zeta_{\epsilon, s}(K),\left(c, c^{\prime}\right)=$ $(a-\nu, a+\rho+\nu)$ (see [L13, 3.1] and 2.8), we deduce that we have canonically

$$
\underline{\left({ }^{\epsilon} L \bullet\left(\zeta_{\epsilon, s}(K)\right)^{\{a+\rho+\nu\}}\right)^{\{a-\nu\}}}=\underline{\left({ }^{\epsilon} L \bullet \zeta_{\epsilon, s}(K)\right)^{\{2 a+\rho\}}} .
$$

We now combine (b) and (c) with Proposition 2.6; we obtain the isomorphism (a). 
2.10. Define $c: G_{\epsilon, s} \times \mathcal{B}^{2} \rightarrow \mathcal{B}^{2}$ by $\left(F, B, B^{\prime}\right) \mapsto\left(F(B), F\left(B^{\prime}\right)\right)$. We show that for $K \in \mathcal{C} G_{\epsilon, s}$ we have canonically

(a)

$$
c^{*} \zeta_{\epsilon, s} K=\overline{\mathbf{Q}}_{l} \otimes \zeta_{\epsilon, s} K .
$$

We have a commutative diagram with cartesian left squares:

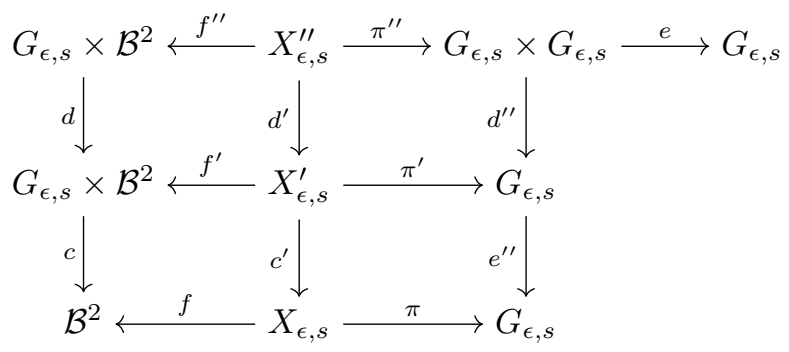

where $f, g$ are as in 1.3 ,

$$
\begin{aligned}
& X_{\epsilon, s}^{\prime}=\left\{\left(\tilde{F}, B, B^{\prime}, F\right) \in G_{\epsilon, s} \times \mathcal{B} \times \mathcal{B} \times G_{\epsilon, s} ; F \tilde{F}(B)=\tilde{F}\left(B^{\prime}\right)\right\}, \\
& X_{\epsilon, s}^{\prime \prime}=\left\{\left(\tilde{F}, B, B^{\prime}, F\right) \in G_{\epsilon, s} \times \mathcal{B} \times \mathcal{B} \times G_{\epsilon, s} ; F(B)=B^{\prime}\right\}, \\
& f^{\prime}\left(\tilde{F}, B, B^{\prime}, F\right)=\left(\tilde{F}, B, B^{\prime}\right), f^{\prime \prime}\left(\tilde{F}, B, B^{\prime}, F\right)=\left(\tilde{F}, B, B^{\prime}\right), \\
& \pi^{\prime}\left(\tilde{F}, B, B^{\prime}, F\right)=F, \pi^{\prime \prime}\left(\tilde{F}, B, B^{\prime}, F\right)=(\tilde{F}, F), \\
& c^{\prime}\left(\tilde{F}, B, B^{\prime}, F\right)=\left(F, \tilde{F}(B), \tilde{F}\left(B^{\prime}\right)\right), c^{\prime \prime}(F)=F, d\left(\tilde{F}, B, B^{\prime}\right)=\left(\tilde{F}, B, B^{\prime}\right), \\
& d^{\prime}\left(\tilde{F}, B, B^{\prime}, F\right)=\left(\tilde{F}, \tilde{F}^{-1} F \tilde{F}, B, B^{\prime}\right), d^{\prime \prime}(\tilde{F}, F)=\tilde{F}^{-1} F \tilde{F}, e(\tilde{F}, F)=F .
\end{aligned}
$$

It is enough to show that $d^{*} f_{!}^{\prime} c^{\prime *} \pi^{*} K=f_{!}^{\prime \prime} \pi^{\prime \prime *} e^{*} K$, or that $f_{!}^{\prime \prime} d^{\prime *} \pi^{\prime *} K=f_{!}^{\prime \prime} \pi^{\prime \prime *} e^{*} K$. It is enough to show that $d^{\prime *} \pi^{\prime *} K=\pi^{\prime \prime *} e^{*} K$, or that $\pi^{\prime \prime *} d^{\prime \prime *} K=\pi^{\prime \prime *} e^{*} K$. Hence it is enough to show that $d^{\prime \prime *} K=e^{*} K$. We identify $G \times G_{\epsilon, s} \leftrightarrow G_{\epsilon, s} \times G_{\epsilon, s}$ by $(g, F) \leftrightarrow(F \operatorname{Ad}(g), F)$. Then $d^{\prime \prime}, e: G_{\epsilon, s} \times G_{\epsilon, s} \rightarrow G_{\epsilon, s}$ become the maps $d_{1}, e_{1}: G \times G_{\epsilon, s} \rightarrow G_{\epsilon, s}$ given by $(g, F) \mapsto \operatorname{Ad}(g)^{-1} F \operatorname{Ad}(g),(g, F)=F$, respectively, and we have $d_{1}^{*} K=e_{1}^{*} K$ by the $G$-equivariance of $K$. Hence $d^{\prime \prime *} K=e^{*} K$ as required.

Using (a) and the definitions we see that for any $K \in \mathcal{C}_{0}^{\mathbf{c}} G_{\epsilon, s}$ we have canonically

$$
c^{*} \underline{\zeta}_{\epsilon, s} K=\overline{\mathbf{Q}}_{l} \otimes \underline{\zeta}_{\epsilon, s} K .
$$

From the definitions (see 1.6) for any $L \in \mathcal{C}_{0}^{\boldsymbol{\oplus}} \mathcal{B}^{2}$ we have $c^{*} L=\overline{\mathbf{Q}}_{l} \otimes^{\epsilon} L$. Comparing with (b) we deduce that we have canonically

$$
\epsilon\left(\underline{\zeta}_{\epsilon, s} K\right)=\underline{\zeta}_{\epsilon, s} K
$$

for any $K \in \mathcal{C}_{0}^{\mathbf{c}} G_{\epsilon, s}$.

\section{Truncated convolution from $G_{\epsilon, s} \times G_{\epsilon^{\prime}, s^{\prime}}$ TO $G_{\epsilon \epsilon^{\prime}, s+s^{\prime}}$}

3.1. Let $\epsilon, \epsilon^{\prime} \in \mathfrak{A}, s, s^{\prime} \in \mathbf{Z}$. We define $\mu: G_{\epsilon, s} \times G_{\epsilon^{\prime}, s^{\prime}} \rightarrow G_{\epsilon \epsilon^{\prime}, s+s^{\prime}}$ by $\left(F, F^{\prime}\right)=$ $F F^{\prime}$ (composition of maps $G \rightarrow G$ ); this is a quasi-morphism; see 1.3. For $K \in$ $\mathcal{D}_{m}\left(G_{\epsilon, s}\right), K^{\prime} \in \mathcal{D}_{m}\left(G_{\epsilon^{\prime}, s^{\prime}}\right)$ we define the convolution $K * K^{\prime} \in \mathcal{D}_{m}\left(G_{\epsilon \epsilon^{\prime}, s+s^{\prime}}\right)$ by $K * K^{\prime}=\mu_{!}\left(K \otimes K^{\prime}\right)$. If $\epsilon^{\prime \prime} \in \mathfrak{A}, s^{\prime \prime} \in \mathbf{Z}$, then for $K, K^{\prime}$ as above and $K^{\prime \prime} \in$ $\mathcal{D}_{m}\left(G_{\epsilon^{\prime \prime}, s^{\prime \prime}}\right)$, we have canonically $\left(K * K^{\prime}\right) * K^{\prime \prime}=K *\left(K^{\prime} * K^{\prime \prime}\right) \in \mathcal{D}_{m}\left(G_{\epsilon \epsilon^{\prime} \epsilon^{\prime \prime}, s+s^{\prime}+s^{\prime \prime}}\right)$ (and we denote this by $K * K^{\prime} * K^{\prime \prime}$ ).

Lemma 3.2. Let $\epsilon, \epsilon^{\prime} \in \mathfrak{A}, s, s^{\prime} \in \mathbf{Z}$. Let $K \in \mathcal{D}_{m}\left(G_{\epsilon, s}\right), L \in \mathcal{D}_{m}\left(\mathcal{B}^{2}\right)$. We have canonically $K * \chi_{\epsilon^{\prime}, s^{\prime}}(L)=\chi_{\epsilon \epsilon^{\prime}, s+s^{\prime}}\left(L \bullet \zeta_{\epsilon, s}(K)\right)$. 
Let

$$
Z=\left\{\left(F_{1}, F_{2}, B, B^{\prime}\right) \in G_{\epsilon, s} \times G_{\epsilon^{\prime}, s^{\prime}} \times \mathcal{B} \times \mathcal{B} ; F_{2}(B)=B^{\prime}\right\}
$$

Define $c: Z \rightarrow G_{\epsilon, s} \times \mathcal{B}^{2}$ by $\left(F_{1}, F_{2}, B, B^{\prime}\right) \mapsto\left(F_{1},\left(B, B^{\prime}\right)\right)$ and $d: Z \rightarrow G_{\epsilon \epsilon^{\prime}, s+s^{\prime}}$ by $\left(F_{1}, F_{2}, B, B^{\prime}\right) \mapsto F_{1} F_{2}$. From the definitions we see that both

$$
K * \chi_{\epsilon^{\prime}, s^{\prime}}(L), \chi_{\epsilon \epsilon^{\prime}, s+s^{\prime}}\left(L \bullet \zeta_{\epsilon, s}(K)\right)
$$

can be identified with $d_{1} c^{*}(K \otimes L)$. The lemma follows. (In the case where $\epsilon=\epsilon^{\prime}=1$ and $s=s^{\prime}=0$ this reduces to [L13, 4.2].)

Proposition 3.3. Let $\epsilon, \epsilon^{\prime} \in \mathfrak{A}, s, s^{\prime} \in \mathbf{Z}$. For any $L, L^{\prime} \in \mathcal{D}_{m}\left(\mathcal{B}^{2}\right)$ we have

$$
\begin{aligned}
& \chi_{\epsilon, s}(L) * \chi_{\epsilon^{\prime}, s^{\prime}}\left(L^{\prime}\right)[[2 \rho+2 \nu]] \\
& \approx\left\{\chi_{\epsilon \epsilon^{\prime}, s+s^{\prime}}\left(L^{\prime} \bullet L_{y} \bullet L \bullet L_{\epsilon(y)^{-1}}\right)[[2|y|]] \otimes \Lambda^{d} \mathcal{X}[[d]](d / 2) ; d \in[0, \rho], y \in W\right\} .
\end{aligned}
$$

From 2.2(b) we deduce

$$
\begin{aligned}
& L^{\prime} \bullet \zeta_{\epsilon, s}\left(\chi_{\epsilon, s}(L)\right)[[2 \nu+2 \rho]] \\
& \approx\left\{L^{\prime} \bullet L_{y} \bullet L \bullet L_{\epsilon(y)^{-1}}[[2|y|]] \otimes \Lambda^{d} \mathcal{X}[[d]](d / 2) ; y \in W, d \in[0, \rho]\right\}
\end{aligned}
$$

and

$$
\begin{aligned}
& \chi_{\epsilon \epsilon^{\prime}, s+s^{\prime}}\left(L^{\prime} \bullet \zeta_{\epsilon, s}\left(\chi_{\epsilon, s}(L)\right)\right)[[2 \nu+2 \rho]] \\
& \approx\left\{\chi_{\epsilon \epsilon^{\prime}, s+s^{\prime}}\left(L^{\prime} \bullet L_{y} \bullet L \bullet L_{\epsilon(y)^{-1}}\right)[[2|y|]] \otimes \Lambda^{d} \mathcal{X}[[d]](d / 2) ; y \in W, d \in[0, \rho]\right\} .
\end{aligned}
$$

It remains to show that $\chi_{\epsilon \epsilon^{\prime}, s+s^{\prime}}\left(L^{\prime} \bullet \zeta_{\epsilon, s}\left(\chi_{\epsilon, s}(L)\right)\right)=\chi_{\epsilon, s}(L) * \chi_{\epsilon^{\prime}, s^{\prime}}\left(L^{\prime}\right)$. This follows from Lemma 3.2 with $K, L$ replaced by $\chi_{\epsilon, s}(L), L^{\prime}$.

In the remainder of this section we fix a two-sided cell $\mathbf{c}$ of $W$; we set $a=\mathbf{a}(\mathbf{c})$.

Proposition 3.4. Let $\epsilon, \epsilon^{\prime} \in \mathfrak{A}, s, s^{\prime} \in \mathbf{Z}$. Assume that $\epsilon(\mathbf{c})=\mathbf{c}, \epsilon^{\prime}(\mathbf{c})=\mathbf{c}$. Let $w, w^{\prime} \in W$ and let $j \in \mathbf{Z}$. We set $C=R_{\epsilon, s, w} * R_{\epsilon^{\prime}, s^{\prime}, w^{\prime}}\left[\left[2 \rho+2 \nu+|w|+\left|w^{\prime}\right|\right]\right] \in$ $\mathcal{D}_{m}\left(G_{\epsilon \epsilon^{\prime}, s+s^{\prime}}\right)$.

(a) If $w \preceq \mathbf{c}$ or $w^{\prime} \preceq \mathbf{c}$, then $C^{j} \in \mathcal{M} \preceq G_{\epsilon \epsilon^{\prime}, s+s^{\prime}}$.

(b) If $j>\Delta+4 a$ and either $w \in \mathbf{c}$ or $w^{\prime} \in \mathbf{c}$, then $C^{j} \in \mathcal{M}^{\prec} G_{\epsilon \epsilon^{\prime}, s+s^{\prime}}$.

(c) If $w \prec \mathbf{c}$ or $w^{\prime} \prec \mathbf{c}$, then $C^{j} \in \mathcal{M}^{\prec} G_{\epsilon \epsilon^{\prime}, s+s^{\prime}}$.

(d) $C^{j}$ is mixed of weight $\leq j$.

(e) If $j \neq \Delta+4 a$ and either $w \in \mathbf{c}$ or $w^{\prime} \in \mathbf{c}$, then $g r_{\Delta+4 a} C^{j} \in \mathcal{M}^{\prec} G_{\epsilon \epsilon^{\prime}, s+s^{\prime}}$.

(f) If $k>\Delta+4 a$ and $w \in \mathbf{c}$ or $w^{\prime} \in \mathbf{c}$, then $g_{k} C^{j} \in \mathcal{M}^{\prec} G_{\epsilon \epsilon^{\prime}, s+s^{\prime}}$.

When $\epsilon=\epsilon^{\prime}=1, s=s^{\prime}=0$, this is just [L13, 4.4]. The proof in the general case is entirely similar; it uses Proposition 3.3 and Lemma 1.4(d),(e).

Proposition 3.5. Let $\epsilon, \epsilon^{\prime} \in \mathfrak{A}, s, s^{\prime} \in \mathbf{Z}$. Assume that $\epsilon(\mathbf{c})=\mathbf{c}, \epsilon^{\prime}(\mathbf{c})=\mathbf{c}$. Let $K \in \mathcal{D}_{m}^{\boldsymbol{中}}\left(G_{\epsilon, s}\right), K^{\prime} \in \mathcal{D}_{m}^{\boldsymbol{中}}\left(G_{\epsilon^{\prime}, s^{\prime}}\right)$.

(a) If $K \in \mathcal{D} \preceq G_{\epsilon, s}$ or $K^{\prime} \in \mathcal{D} \preceq G_{\epsilon^{\prime}, s^{\prime}}$, then $K * K^{\prime} \in \mathcal{D} \preceq G_{\epsilon \epsilon^{\prime}, s+s^{\prime}}$; if $K \in \mathcal{D}^{\prec} G_{\epsilon, s}$ or $K^{\prime} \in \mathcal{D}^{\prec} G_{\epsilon^{\prime}, s^{\prime}}$, then $K * K^{\prime} \in \mathcal{D}^{\prec} G_{\epsilon \epsilon^{\prime}, s+s^{\prime}}$.

(b) If $K \in \mathcal{M}^{\preceq} G_{\epsilon, s}, K^{\prime} \in \mathcal{M} \preceq G_{\epsilon^{\prime}, s^{\prime}}$ and $j>\rho+2 a$, then $\left(K * K^{\prime}\right)^{j} \in$ $\mathcal{M}^{\prec} G_{\epsilon \epsilon^{\prime}, s+s^{\prime}}$.

When $\epsilon=\epsilon^{\prime}=1, s=s^{\prime}=0$, this is just [L13, 4.5]. The proof in the general case is entirely similar. It is enough to prove the proposition assuming in addition that $K=A \in C S\left(G_{\epsilon, s}\right), K^{\prime}=A^{\prime} \in C S\left(G_{\epsilon^{\prime}, s^{\prime}}\right)$. By 1.5(b), we can find $w \in \mathbf{c}_{A}$, $w^{\prime} \in \mathbf{c}_{A^{\prime}}$ such that $\left(A: R_{\epsilon, s, w}^{n_{w}}\right) \neq 0,\left(A^{\prime}: R_{\epsilon^{\prime}, s^{\prime}, w^{\prime}}^{n_{w^{\prime}}}\right) \neq 0$. Then $A\left[-n_{w}\right]$ is a direct summand of $R_{\epsilon, s, w}$ and $A^{\prime}\left[-n_{w^{\prime}}\right]$ is a direct summand of $R_{\epsilon^{\prime}, s^{\prime}, w^{\prime}}$. Hence $A * A^{\prime}$ is 
a direct summand of $R_{\epsilon, s, w} * R_{\epsilon^{\prime}, s^{\prime}, w^{\prime}}\left[2 \Delta+\mathbf{a}(w)+\mathbf{a}\left(w^{\prime}\right)+|w|+\left|w^{\prime}\right|\right]$ and $\left(A * A^{\prime}\right)^{j}$ is a direct summand of

$$
\left(R_{\epsilon, s, w} * R_{\epsilon^{\prime}, s^{\prime}, w^{\prime}}\left[2 \rho+2 \nu+|w|+\left|w^{\prime}\right|\right]\right)^{j+\mathbf{a}(w)+\mathbf{a}\left(w^{\prime}\right)+2 \nu} .
$$

Using Proposition 3.4 we deduce that (a) holds and that $\left(A * A^{\prime}\right)^{j} \in \mathcal{M}^{\prec} G$ provided that $j+\mathbf{a}(w)+\mathbf{a}\left(w^{\prime}\right)+2 \nu>\Delta+4 a$. Hence (b) holds. (To prove (b) we can assume, by (a), that $w \in \mathbf{c}, w^{\prime} \in \mathbf{c}$ hence $\mathbf{a}(w)=\mathbf{a}\left(w^{\prime}\right)=a$.)

3.6. Let $\epsilon, \epsilon^{\prime} \in \mathfrak{A}, s, s^{\prime} \in \mathbf{Z}$. Assume that $\epsilon(\mathbf{c})=\mathbf{c}, \epsilon^{\prime}(\mathbf{c})=\mathbf{c}$. For $K \in \mathcal{C}_{0}^{\mathbf{c}} G_{\epsilon, s}$, $K^{\prime} \in \mathcal{C}_{0}^{\mathbf{c}} G_{\epsilon^{\prime}, s^{\prime}}$ we set

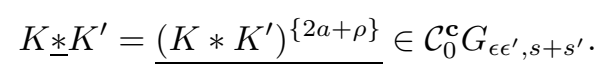

We say that $K * K^{\prime}$ is the truncated convolution of $K, K^{\prime}$.

Proposition 3.7. Let $\epsilon, \epsilon^{\prime}, \epsilon^{\prime \prime} \in \mathfrak{A}, s, s^{\prime}, s^{\prime \prime} \in \mathbf{Z}$. Assume that $\epsilon(\mathbf{c})=\mathbf{c}, \epsilon^{\prime}(\mathbf{c})=\mathbf{c}$, $\epsilon^{\prime \prime}(\mathbf{c})=\mathbf{c}$. Let $K, K^{\prime}, K^{\prime \prime}$ be in $\mathcal{C}_{0}^{\mathbf{c}} G_{\epsilon, s}, \mathcal{C}_{0}^{\mathbf{c}} G_{\epsilon^{\prime}, s^{\prime}}, \mathcal{C}_{0}^{\mathbf{c}} G_{\epsilon^{\prime \prime}, s^{\prime \prime}}$, respectively. There is a canonical isomorphism

$$
\left(K \underline{*} K^{\prime}\right) \underline{*} K^{\prime \prime} \stackrel{\sim}{\longrightarrow} K \underline{*}\left(K^{\prime} \underline{*} K^{\prime \prime}\right) .
$$

When $\epsilon=\epsilon^{\prime}=\epsilon^{\prime \prime}=1, s=s^{\prime}=s^{\prime \prime}=0$, this is just [L13, 4.7]. The proof in the general case is entirely similar; it uses Lemma 1.9 and Proposition 3.5.

Proposition 3.8. Let $\epsilon, \epsilon^{\prime} \in \mathfrak{A}, s, s^{\prime} \in \mathbf{Z}$. Assume that $\epsilon(\mathbf{c})=\mathbf{c}, \epsilon^{\prime}(\mathbf{c})=\mathbf{c}$. Let $K \in \mathcal{C}_{0}^{\mathbf{c}} G_{\epsilon, s}, K^{\prime} \in \mathcal{C}_{0}^{\mathbf{c}} G_{\epsilon^{\prime}, s^{\prime}}$. There is a canonical isomorphism (in $\mathcal{C}_{0}^{\mathbf{c}} \mathcal{B}^{2}$ ):

$$
\underline{\zeta}_{\epsilon^{\prime}, s^{\prime}}\left(K^{\prime}\right) \underline{\zeta}_{\epsilon, s}(K) \stackrel{\sim}{\longrightarrow} \underline{\zeta}_{\epsilon \epsilon^{\prime}, s+s^{\prime}}\left(K * K^{\prime}\right) .
$$

When $\epsilon=\epsilon^{\prime}=1, s=s^{\prime}=0$ this is just [L13, 5.2]. In the general case, we first show that for any $\mathfrak{K} \in \mathcal{D}_{m}\left(G_{\epsilon, s}\right), \mathfrak{K}^{\prime} \in \mathcal{D}_{m}\left(G_{\epsilon^{\prime}, s^{\prime}}\right)$ we have canonically

$$
\zeta_{\epsilon^{\prime}, s^{\prime}}\left(\mathfrak{K}^{\prime}\right) \bullet \zeta_{\epsilon, s}(\mathfrak{K})=\zeta_{\epsilon \epsilon^{\prime}, s+s^{\prime}}\left(\mathfrak{K} * \mathfrak{K}^{\prime}\right) .
$$

(When $\epsilon=\epsilon^{\prime}=1, s=s^{\prime}=0$ this is just [L13, 5.5], which goes back to the work of Ginzburg.) Let

$$
Y=\left\{\left(B_{1}, B_{2}, F_{1}, F_{2}\right) \in \mathcal{B} \times \mathcal{B} \times G_{\epsilon, s} \times G_{\epsilon^{\prime}, s^{\prime}} ; F_{1} F_{2}\left(B_{1}\right)=B_{2}\right\} .
$$

Define $f^{\prime}: Y \rightarrow \mathcal{B} \times \mathcal{B}$ by $f^{\prime}\left(B_{1}, B_{2}, F_{1}, F_{2}\right)=\left(B_{1}, B_{2}\right)$. Define $\pi^{\prime}: Y \rightarrow G_{\epsilon, s} \times G_{\epsilon^{\prime}, s^{\prime}}$ by $\pi^{\prime}\left(B_{1}, B_{2}, F_{1}, F_{2}\right)=\left(F_{1}, F_{2}\right)$. From the definitions we see that $f_{!}^{\prime} \pi^{\prime *}\left(\mathfrak{K} \otimes \mathfrak{K}^{\prime}\right)$ is canonically isomorphic to $\zeta_{\epsilon^{\prime}, s^{\prime}}\left(\mathfrak{K}^{\prime}\right) \bullet \zeta_{\epsilon, s}(\mathfrak{K})$ and to $\zeta_{\epsilon \epsilon^{\prime}, s+s^{\prime}}\left(\mathfrak{K} * \mathfrak{K}^{\prime}\right)$. Thus (a) holds. Now the lemma follows from (a), using Lemma 1.9, by an argument similar to that in [L13, 5.6].

\section{Analysis of the Composition $\underline{\zeta}_{\epsilon, s} \underline{\chi}_{\epsilon, s}$}

4.1. In the remainder of this paper we fix a two-sided cell $\mathbf{c}$ of $W$; we set $a=\mathbf{a}(\mathbf{c})$. We also fix $\epsilon \in \mathfrak{A}$ such that $\epsilon(\mathbf{c})=\mathbf{c}$. In this section we fix $s \in \mathbf{Z}$. Let $e, f, e^{\prime}$ be integers such that $e \leq f \leq e^{\prime}-3$ and let $\mathbf{e}=e^{\prime}-e+1$; we have $\mathbf{e} \geq 4$. We set

$$
\mathcal{Y}=\left\{\left(\left(B_{e}, B_{e+1}, \ldots, B_{e^{\prime}}\right), F\right) \in \mathcal{B}^{\mathbf{e}} \times G_{\epsilon, s} ; F\left(B_{f}\right)=B_{f+3}, F\left(B_{f+1}\right)=B_{f+2}\right\} .
$$

Define $\vartheta: \mathcal{Y} \rightarrow \mathcal{B}^{\text {e }}$ by $\left(\left(B_{e}, B_{e+1}, \ldots, B_{e^{\prime}}\right), F\right) \mapsto\left(B_{e}, B_{e+1}, \ldots, B_{e^{\prime}}\right)$. For $i, j$ in $\left\{e, e+1, \ldots, e^{\prime}\right\}$ let $p_{i j}: \mathcal{B}^{e} \rightarrow \mathcal{B}^{2}$ be the projection to the $i, j$ coordinate; define 
$h_{i j}: \mathcal{Y} \rightarrow \mathcal{B}^{2}$ by $h_{i j}=p_{i j} \vartheta$. Now $G^{\mathbf{e}-2}$ acts on $\mathcal{Y}$ by

$$
\begin{aligned}
& \left(g_{e}, \ldots, g_{f}, g_{f+3}, \ldots, g_{e^{\prime}}\right):\left(\left(B_{e}, B_{e+1}, \ldots, B_{e^{\prime}}\right), F\right) \mapsto \\
& \left(\operatorname{Ad}\left(g_{e}\right)\left(B_{e}\right), \operatorname{Ad}\left(g _ { e + 1 } \left(\left(B_{e+1}\right), \ldots, \operatorname{Ad}\left(g_{f-1}\right)\left(B_{f-1}\right), \operatorname{Ad}\left(g_{f}\right)\left(B_{f}\right), \operatorname{Ad}\left(g_{f}\right)\left(B_{f+1}\right),\right.\right.\right. \\
& \left.\operatorname{Ad}\left(g_{f+3}\right)\left(B_{f+2}\right), \operatorname{Ad}\left(g_{f+3}\right)\left(B_{f+3}\right), \operatorname{Ad}\left(g_{f+4}\right)\left(B_{f+4}\right), \ldots, \operatorname{Ad}\left(g_{e^{\prime}}\right)\left(B_{e^{\prime}}\right)\right), \\
& \left.\operatorname{Ad}\left(g_{f+3}\right) F \operatorname{Ad}\left(g_{f}^{-1}\right)\right) ;
\end{aligned}
$$

this induces a $G^{\mathbf{e}-2}$-action on $\mathcal{B}^{\mathbf{e}}$ so that $\vartheta$ is $G^{\mathbf{e}-2}$-equivariant.

Let $E=\left\{e, e+1, \ldots, e^{\prime}-1\right\}-\{f, f+2\}$. Assume that $x_{n} \in \mathbf{c}$ are given for $n \in E$. Let $P=\bigotimes_{n \in E} p_{n, n+1}^{*} \mathbf{L}_{x_{n}} \in \mathcal{D}_{m} \mathcal{B}^{\mathbf{e}}, \tilde{P}=\bigotimes_{n \in E} h_{n, n+1}^{*} \mathbf{L}_{x_{n}}=\vartheta^{*} P \in \mathcal{D}_{m} \mathcal{Y}$. In $4.1-4.7$ we will study

$$
h_{e e^{\prime} !} \tilde{P} \in \mathcal{D}_{m} \mathcal{B}^{2} .
$$

Setting $\Xi=\vartheta_{!} \overline{\mathbf{Q}}_{l} \in \mathcal{D}_{m} \mathcal{B}^{\mathbf{e}}$, we have

$$
h_{e e^{\prime} !} \tilde{P}=p_{e e !}(\Xi \otimes P) .
$$

Clearly, $\Xi^{j}$ is $G^{\mathbf{e}-2}$-equivariant for any $j$. For any $y, y^{\prime}$ in $W$ we set

$$
Z_{y, y^{\prime}}:=\left\{\left(B_{e}, B_{e+1}, \ldots, B_{e^{\prime}}\right) \in \mathcal{B}^{\mathbf{e}} ;\left(B_{f}, B_{f+1}\right) \in \mathcal{O}_{y},\left(B_{f+2}, B_{f+3}\right) \in \mathcal{O}_{y^{\prime}}\right\} .
$$

These are the orbits of the $G^{\mathbf{e}-2}$-action on $\mathcal{B}^{\mathbf{e}}$. Note that the fibre of $\vartheta$ over a point of $Z_{y, y^{\prime}}$ is isomorphic to $T \times \mathbf{k}^{\nu-|y|}$ if $y^{\prime}=\epsilon(y)^{-1}$ and is empty if $y^{\prime} \neq \epsilon(y)^{-1}$. Thus,

(a) $\left.\Xi\right|_{Z_{y, y^{\prime}}}$ is 0 if $y^{\prime} \neq \epsilon(y)^{-1}$

and for any $y \in W$ we have

(b)

$\left.\mathcal{H}^{h} \Xi\right|_{Z_{y, \epsilon(y)^{-1}}}=0$ if $h>2 \nu-2|y|+2 \rho,\left.\quad \mathcal{H}^{2 \nu-2|y|+2 \rho} \Xi\right|_{Z_{y, \epsilon(y)-1}}=\overline{\mathbf{Q}}_{l}(-\nu+|y|-\rho)$.

The closure of $Z_{y, y^{\prime}}$ in $\mathcal{B}^{\mathbf{e}}$ is denoted by $\bar{Z}_{y, y^{\prime}}$. We set $k_{\mathbf{e}}=\mathbf{e} \nu+2 \rho$. We have the following result.

Lemma 4.2. (a) We have $\Xi^{j}=0$ for any $j>k_{\mathbf{e}}$. Hence, setting $\Xi^{\prime}=\tau_{\leq k_{\mathbf{e}}-1} \Xi$, we have a canonical distinguished triangle $\left(\Xi^{\prime}, \Xi, \Xi^{k_{\mathbf{e}}}\left[-k_{\mathbf{e}}\right]\right)$.

(b) If $\xi \in Z_{y, y^{\prime}}$ and $i=2 \nu-|y|-\left|y^{\prime}\right|+2 \rho$, the induced homomorphism $\mathcal{H}_{\xi}^{i} \Xi \rightarrow$ $\mathcal{H}_{\xi}^{i-k_{\mathbf{e}}}\left(\Xi^{k_{\mathbf{e}}}\right)$ is an isomorphism.

When $\epsilon=1, s=0$ this is just [L13, 6.2]. The proof in the general case is entirely similar; it uses $4.1(\mathrm{a}),(\mathrm{b})$.

4.3. For any $y, y^{\prime}$ in $W$ let $\mathfrak{T}_{y, y^{\prime}}$ be the intersection cohomology complex of $\bar{Z}_{y, y^{\prime}}$ extended by 0 on $\mathcal{B}^{\mathbf{e}}-\bar{Z}_{y, y^{\prime}}$, to which $\left[\left[(\mathbf{e}-2) \nu+|y|+\left|y^{\prime}\right|\right]\right]$ is applied. It is a pure perverse sheaf of weight 0 . Note that

$$
\mathfrak{T}_{y, y^{\prime}}=p_{f, f+1}^{*} \mathbf{L}_{y} \otimes p_{f+2, f+3}^{*} \mathbf{L}_{y^{\prime}}[[(\mathbf{e}-4) \nu]] .
$$

We have the following result.

Lemma 4.4. We have canonically $\operatorname{gr}_{0}\left(\Xi^{k_{\mathbf{e}}}\left(k_{\mathbf{e}} / 2\right)\right)=\bigoplus_{y \in W} \mathfrak{T}_{y, \epsilon(y)^{-1}}$.

When $\epsilon=1, s=0$ this is just [L13, 6.4]. The proof in the general case is entirely similar; it uses Lemma 4.2(b) and 4.1. 
4.5. Let $y, \tilde{y} \in W$. Using the definitions and 1.2 (a) we have

$$
\begin{aligned}
& p_{e e^{\prime} !}\left(\mathfrak{T}_{y, \tilde{y}} \otimes P[[(6-2 \mathbf{e}) \nu]]\right) \\
& =L_{x_{1}}^{\sharp} \bullet L_{x_{f-1}}^{\sharp} L_{y}^{\sharp} L_{x_{f+1}}^{\sharp} L_{\tilde{y}}^{\sharp} L_{x_{f+3}}^{\sharp} L_{x_{e^{\prime}}}^{\sharp}\left[\left[\nu+|y|+|\tilde{y}|+\sum_{n \in E}\left|x_{n}\right|\right]\right] .
\end{aligned}
$$

Lemma 4.6. The map $\Xi \rightarrow \Xi^{k_{\mathbf{e}}}\left[-k_{\mathbf{e}}\right]$ (coming from $\left(\Xi^{\prime}, \Xi, \Xi^{k_{\mathbf{e}}}\left[-k_{\mathbf{e}}\right]\right.$ ) in Lemma 4.2(a)) induces a morphism

$$
\left(p_{e e^{\prime} !}(\Xi \otimes P)\right)^{(\mathbf{e}-2) a+(6-\mathbf{e}) \nu+2 \rho} \rightarrow\left(p_{e e^{\prime} !}\left(\Xi^{k_{\mathbf{e}}} \otimes P\right)\right)^{(\mathbf{e}-2) a+(6-\mathbf{e}) \nu+2 \rho-k_{\mathbf{e}}}
$$

whose kernel and cokernel are in $\mathcal{M}_{m}^{\prec} \mathcal{B}^{2}$.

When $\epsilon=1, s=0$ this is just [L13, 6.6]. The proof in the general case is entirely similar; it uses Lemma 4.5(a) and [L13, 2.2(a)].

Lemma 4.7. We have canonically

$$
\underline{\left(h_{e e^{\prime} !} \tilde{P}\right)^{\{(\mathbf{e}-2) a+(6-\mathbf{e}) \nu+2 \rho\}}}=\bigoplus_{y \in \mathbf{c}} Q_{y}
$$

where

$$
\begin{aligned}
Q_{y} & =\frac{\left(p_{e e^{\prime} !}\left(\mathfrak{T}_{y, \epsilon(y)^{-1}} \otimes P\right)\right)^{\{(\mathbf{e}-2) a+(6-2 \mathbf{e}) \nu\}}}{} \\
& =\mathbf{L}_{x_{1}} \mathbf{L}_{x_{f-1}} \mathbf{L}_{y} \mathbf{L}_{x_{f+1}} \mathbf{L}_{\epsilon(y)^{-1}} \mathbf{L}_{x_{f+3}} \mathbf{L}_{x_{e^{\prime}}}
\end{aligned}
$$

When $\epsilon=1, s=0$ this is just [L13, 6.7]. The proof in the general case is entirely similar; it uses 4.6, 4.5(a) and [L13, 2.2(a), 2.3, 3.2].

Theorem 4.8. Let $x \in \mathbf{c}$. We have canonically

$$
\underline{\zeta}_{\epsilon, s}\left(\underline{\chi}_{\epsilon, s}\left(\mathbf{L}_{x}\right)\right)=\bigoplus_{y \in \mathbf{c}} \mathbf{L}_{y} \bullet \mathbf{L}_{x} \bullet \mathbf{L}_{\epsilon(y)^{-1}}
$$

When $\epsilon=1, s=0$ this is just [L13, 6.8]. The proof in the general case is entirely similar; it uses Lemma 4.7, the proofs of Propositions 2.2 and 2.7(b).

4.9. Using [L13, 2.4] we see that Theorem 4.8(a) implies

$$
\underline{\zeta}_{\epsilon, s} \underline{\chi}_{\epsilon, s} \mathbf{L}_{x} \cong \bigoplus_{z \in \mathbf{c}}\left(\mathbf{L}_{z}\right)^{\oplus \psi_{x}(z)}
$$

in $\mathcal{C}^{\mathbf{c}} \mathcal{B}^{2}$ where $\psi_{x}(z) \in \mathbf{N}$ are given by the following equation in $\mathbf{J}^{\mathbf{c}}$ :

$$
\sum_{y \in \mathbf{c}} t_{y} t_{x} t_{\epsilon(y)^{-1}}=\sum_{z \in \mathbf{c}} \psi_{x}(z) t_{z}
$$

\section{Adjunction formula (Weak form)}

Proposition 5.1. Let $\epsilon^{\prime} \in \mathfrak{A}, s, s^{\prime} \in \mathbf{Z}$. We assume that $\epsilon^{\prime}(\mathbf{c})=\mathbf{c}$. Let $K \in$ $\mathcal{C}_{0}^{\mathbf{c}}\left(G_{\epsilon, s}\right), L \in \mathcal{C}_{0}^{\mathbf{c}}\left(\mathcal{B}^{2}\right)$. We have canonically

$$
K \underline{x}_{\epsilon^{\prime}, s^{\prime}}(L)=\underline{\chi}_{\epsilon \epsilon^{\prime}, s+s^{\prime}}\left(L \underline{\bullet}_{\epsilon, s}(K)\right) .
$$

When $\epsilon=\epsilon^{\prime}=1, s=s^{\prime}=0$ this is just [L13, 8.1]. The proof in the general case is entirely similar; it uses Lemmas 3.2 and 1.9.

For future use we recall the following result in [L13, 8.7]. (Here $\mathbf{1}^{\prime}$ is as in [L13, 8.6] and $b^{\prime}: \mathcal{B}^{2} \rightarrow \mathcal{B}^{2}$ is given by $\left(B, B^{\prime}\right) \mapsto\left(B^{\prime}, B\right)$, see 1.3.)

(b) Let $L, L^{\prime} \in \mathcal{C}^{\mathbf{c}} \mathcal{B}^{2}$. We have canonically

$$
\operatorname{Hom}_{\mathcal{C}^{\mathrm{c}} \mathcal{B}^{2}}\left(\mathbf{1}^{\prime}, L \bullet L^{\prime}\right)=\operatorname{Hom}_{\mathcal{C}^{\mathrm{c}} \mathcal{B}^{2}}\left(\mathfrak{D}\left(b_{!}^{\prime} L^{\prime}\right), L\right) .
$$


5.2. Let $s \in \mathbf{Z}$. When $\epsilon=1, s=0$, the arguments in this subsection reduce to arguments in [L13, 8.8]. Let $u^{\prime}: G_{\epsilon^{-1},-s} \rightarrow \mathbf{p}$ be the obvious map. From [L2, (7.4.1),(7.4.2)] and its proof we see that if $K, K^{\prime} \in \mathcal{M}_{m}^{\prec} G_{\epsilon^{-1},-s}$ are semisimple, then we have canonically

$$
\left(u_{!}^{\prime}\left(K \otimes K^{\prime}\right)\right)^{0}=\operatorname{Hom}_{\mathcal{M} G_{\epsilon-1,-s}}\left(\mathfrak{D}(K), K^{\prime}\right), \quad\left(u_{!}^{\prime}\left(K \otimes K^{\prime}\right)\right)^{j}=0 \text { if } j>0 .
$$

We deduce that if $K, K^{\prime}$ are also pure of weight 0 , then $\left(u_{!}^{\prime}\left(K \otimes K^{\prime}\right)\right)^{0}$ is pure of weight zero, that is, $\left(u_{!}^{\prime}\left(K \otimes K^{\prime}\right)\right)^{0}=g r_{0}\left(u_{!}^{\prime}\left(K \otimes K^{\prime}\right)\right)^{0}$. Let $\iota: \mathbf{p} \rightarrow G=G_{1,0}$ be the map with image 1. From the definitions we see that we have $u_{!}^{\prime}\left(K \otimes K^{\prime}\right)=\iota^{*}\left(b_{!}(K) *\right.$ $\left.K^{\prime}\right)$ where $b: G_{\epsilon^{-1},-s} \rightarrow G_{\epsilon, s}$ is given by $F \mapsto F^{-1}$. Hence for $K, K^{\prime} \in \mathcal{C}_{0}^{\mathbf{c}} G_{\epsilon^{-1},-s}$ we have

(a) $\quad \operatorname{Hom}_{\mathcal{C}^{\mathrm{c}} G_{\epsilon}-1,-s}\left(\mathfrak{D}(K), K^{\prime}\right)=\left(\iota^{*}\left(b_{!}(K) * K^{\prime}\right)\right)^{0}=\left(\iota^{*}\left(b_{!}(K) * K^{\prime}\right)\right)^{\{0\}}$.

Applying [L13, 8.2] with $\Phi: \mathcal{D}_{\bar{m}}^{\prec} G_{1,0} \rightarrow \mathcal{D}_{m} \mathbf{p}, K_{1} \mapsto \iota^{*} K_{1}, c=-2 a-\rho$ (see [L13, 8.3(a)], $K$ replaced by $b_{!}(K) * K^{\prime} \in \mathcal{D}_{m}\left(G_{1,0}\right)$ and $c^{\prime}=2 a+\rho$ we see that we have canonically

$$
\left(\iota^{*}\left(b_{!}(K) * K^{\prime}\right)\right)^{\{-2 a-\rho\}} \subset\left(\iota^{*}\left(b_{!}(K) * K^{\prime}\right)\right)^{\{0\}} .
$$

In particular, if $L, L^{\prime} \in \mathcal{C}_{0}^{\mathbf{c}} \mathcal{B}^{2}$, then we have canonically

$$
\left(\iota^{*}\left(\underline{\chi}_{\epsilon, s}\left(L^{\prime}\right) * \underline{\chi}_{\epsilon^{-1},-s}(L)\right)\right)^{\{-2 a-\rho\}} \subset\left(\iota^{*}\left(\underline{\chi}_{\epsilon, s}\left(L^{\prime}\right) * \underline{\chi}_{\epsilon^{-1},-s}(L)\right)\right)^{\{0\}} .
$$

Using the equality

$$
\left(\iota^{*}\left(\underline{\chi}_{\epsilon, s}\left(L^{\prime}\right) \underline{*} \underline{\chi}_{\epsilon^{-1},-s}(L)\right)\right)^{\{-2 a-\rho\}}=\left(\iota^{*}\left(\underline{\chi}_{1,0}\left(L \underline{\bullet} \underline{\zeta}_{\epsilon, s}\left(\underline{\chi}_{\epsilon, s}\left(L^{\prime}\right)\right)\right)\right)\right)^{\{-2 a-\rho\}}
$$

which comes from Proposition 5.1, we deduce that we have canonically

$$
\left(\iota^{*}\left(\underline{\chi}_{1,0}\left(L \underline{\bullet} \underline{\zeta}_{\epsilon, s}\left(\underline{\chi}_{\epsilon, s}\left(L^{\prime}\right)\right)\right)\right)\right)^{\{-2 a-\rho\}} \subset\left(\iota^{*}\left(\underline{\chi}_{\epsilon, s}\left(L^{\prime}\right) * \underline{\chi}_{\epsilon^{-1},-s}(L)\right)\right)^{\{0\}}
$$

or equivalently, using (a) with $K, K^{\prime}$ replaced by $b^{*} \underline{\chi}_{\epsilon, s}\left(L^{\prime}\right), \underline{\chi}_{\epsilon^{-1},-s}(L)$ :

$$
\begin{aligned}
& \left(\iota^{*}\left(\underline{\chi}_{1,0}\left(L \underline{\bullet} \underline{\zeta}_{\epsilon, s}\left(\underline{\chi}_{\epsilon, s}\left(L^{\prime}\right)\right)\right)\right)\right)^{\{-2 a-\rho\}} \subset \operatorname{Hom}_{\mathcal{C}^{\mathrm{c}} G_{\epsilon^{-1},-s}}\left(\mathfrak{D}\left(b^{*} \underline{\chi}_{\epsilon, s}\left(L^{\prime}\right)\right), \underline{\chi}_{\epsilon^{-1},-s}(L)\right) \\
& =\operatorname{Hom}_{\mathcal{C}^{\mathbf{c}} G_{\epsilon, s}}\left(\mathfrak{D}\left(b ! \underline{\chi}_{\epsilon^{-1},-s}(L)\right), \underline{\chi}_{\epsilon, s}\left(L^{\prime}\right)\right) .
\end{aligned}
$$

Now using [L13, 8.6(c)], we deduce that we have canonically

$$
\operatorname{Hom}_{\mathcal{C}^{\mathbf{c}} \mathcal{B}^{2}}\left(\mathbf{1}^{\prime}, L \underline{\boldsymbol{\iota}}_{\epsilon, s} \underline{\chi}_{\epsilon, s} L^{\prime}\right) \subset \operatorname{Hom}_{\mathcal{C}^{\mathbf{c}} G_{\epsilon, s}}\left(\mathfrak{D}\left(b ! \underline{\chi}_{\epsilon^{-1},-s}(L), \underline{\chi}_{\epsilon, s}\left(L^{\prime}\right)\right)\right.
$$

or equivalently (see Proposition 5.1(b)):

$$
\operatorname{Hom}_{\mathcal{C}^{\mathbf{c}} \mathcal{B}^{2}}\left(\mathfrak{D}\left(b_{!}^{\prime} L\right), \underline{\zeta}_{\epsilon, s} \underline{\chi}_{\epsilon, s} L^{\prime}\right) \subset \operatorname{Hom}_{\mathcal{C}^{\mathbf{c}} G_{\epsilon, s}}\left(\mathfrak{D}\left(b ! \underline{\chi}_{\epsilon^{-1},-s}(L), \underline{\chi}_{\epsilon, s}\left(L^{\prime}\right)\right)\right.
$$

We now set ' $L=\mathfrak{D}\left(b_{!}^{\prime} L\right)$ and note that

$$
\mathfrak{D}\left(b_{!} \underline{\chi}_{\epsilon^{-1},-s}(L)\right)=\mathfrak{D}\left(\underline{\chi}_{\epsilon, s}\left(b_{!}^{\prime} L\right)\right)=\underline{\chi}_{\epsilon, s}\left(\mathfrak{D}\left(b_{!}^{\prime} L\right)\right)=\underline{\chi}_{\epsilon, s}\left({ }^{\prime} L\right) ;
$$

see $1.3,1.10(\mathrm{a})$. We obtain

$$
\operatorname{Hom}_{\mathcal{C}^{\mathfrak{c}} \mathcal{B}^{2}}\left({ }^{\prime} L, \underline{\zeta}_{\epsilon, s} \underline{\chi}_{\epsilon, s} L^{\prime}\right) \subset \operatorname{Hom}_{\mathcal{C}^{\mathrm{c}} G_{\epsilon, s}}\left(\underline{\chi}_{\epsilon, s}\left({ }^{\prime} L\right), \underline{\chi}_{\epsilon, s}\left(L^{\prime}\right)\right)
$$

for any ${ }^{\prime} L, L^{\prime} \in \mathcal{C}_{0}^{\mathbf{c}} \mathcal{B}^{2}$.

We have the following result which is a weak form of an adjunction formula, of which the full form will be proved in Theorem 6.6. 
Proposition 5.3. Let $s \in \mathbf{Z}$. For any ${ }^{\prime} L, L^{\prime} \in \mathcal{C}_{0}^{\mathbf{c}} \mathcal{B}^{2}$ we have canonically

$$
\operatorname{Hom}_{\mathcal{C}^{\mathrm{c}} \mathcal{B}^{2}}\left({ }^{\prime} L, \underline{\zeta}_{\epsilon, s} \underline{\chi}_{\epsilon, s}\left(L^{\prime}\right)\right)=\operatorname{Hom}_{\mathcal{C}^{\mathrm{c}} G_{\epsilon, s}}\left(\underline{\chi}_{\epsilon, s}\left({ }^{\prime} L\right), \underline{\chi}_{\epsilon, s}\left(L^{\prime}\right)\right)
$$

We can assume that ${ }^{\prime} L=\mathbf{L}_{z}, L^{\prime}=\mathbf{L}_{u}$ where $z, u \in \mathbf{c}$. By 4.9(a) and 1.8(b), both sides of the inclusion Proposition 5.2(b) have dimension $\sum_{y \in \mathbf{c}} \tau\left(t_{y^{-1}} t_{z} t_{\epsilon(y)} t_{u^{-1}}\right)$. Hence that inclusion is an equality. The proposition is proved. (The case where $\epsilon=1, s=0$ is treated in [L13, 8.9].)

\section{Equivalence of $\mathcal{C}^{\mathbf{c}} G_{\epsilon, s}$ With the $\epsilon$-Centre of $\mathcal{C}^{\mathbf{c}} \mathcal{B}^{2}$}

6.1. For $\epsilon^{\prime} \in \mathfrak{A}$ such that $\epsilon^{\prime}(\mathbf{c})=\mathbf{c}$ and $s, s^{\prime} \in \mathbf{Z}$, the bifunctor $\mathcal{C}_{0}^{\mathbf{c}} G_{\epsilon, s} \times \mathcal{C}_{0}^{\mathbf{c}} G_{\epsilon^{\prime}, s^{\prime}} \rightarrow$ $\mathcal{C}_{0}^{\mathbf{c}} G_{\epsilon \epsilon^{\prime}, s+s^{\prime}}, K, K^{\prime} \mapsto K * K^{\prime}$ in 3.6 defines a bifunctor $\mathcal{C}^{\mathbf{c}} G_{\epsilon, s} \times \mathcal{C}^{\mathbf{c}} G_{\epsilon^{\prime}, s^{\prime}} \rightarrow \mathcal{C}^{\mathbf{c}} G_{\epsilon \epsilon^{\prime}, s+s^{\prime}}$ denoted again by $K, K^{\prime} \mapsto K \underline{x} K^{\prime}$ as follows. Let $K \in \mathcal{C}^{\mathbf{c}} G_{\epsilon, s}, K^{\prime} \in \mathcal{C}^{\mathbf{c}} G_{\epsilon^{\prime}, s^{\prime}}$; we choose mixed structures of pure weight 0 on $K, K^{\prime}$ (this is possible if $s_{0}$ in 0.3 is large enough), we define $K \underline{x} K^{\prime} \in \mathcal{C}_{0}^{\mathbf{c}} G_{\epsilon \epsilon^{\prime}, s+s^{\prime}}$ as in 3.6 in terms of these mixed structures and we then disregard the mixed structure on $K * K^{\prime}$. The resulting object of $\mathcal{C}^{\mathbf{c}} G_{\epsilon \epsilon^{\prime}, s+s^{\prime}}$ is denoted again by $K \underline{*} K^{\prime}$; it is independent of the choices made.

In the same way, the bifunctor $\mathcal{C}_{0}^{\mathbf{c}} \mathcal{B}^{2} \times \mathcal{C}_{0}^{\mathbf{c}} \mathcal{B}^{2} \rightarrow \mathcal{C}_{0}^{\mathbf{c}} \mathcal{B}^{2}, L, L^{\prime} \mapsto L \bullet L^{\prime}$ gives rise to a bifunctor $\mathcal{C}^{\mathbf{c}} \mathcal{B}^{2} \times \mathcal{C}^{\mathbf{c}} \mathcal{B}^{2} \rightarrow \mathcal{C}^{\mathbf{c}} \mathcal{B}^{2}$ denoted again by $L, L^{\prime} \mapsto L \bullet L^{\prime}$; the functor $\underline{\chi}_{\epsilon, s}: \mathcal{C}_{0}^{\mathbf{c}} \mathcal{B}^{2} \rightarrow \mathcal{C}_{0}^{\mathbf{c}} G_{\epsilon, s}$ gives rise to a functor $\mathcal{C}^{\mathbf{c}} \mathcal{B}^{2} \rightarrow \mathcal{C}^{\mathbf{c}} G_{s}$ denoted again by $\underline{\chi}_{\epsilon, s}$ (it is again called truncated induction); the functor $\underline{\zeta}_{\epsilon, s}: \mathcal{C}_{0}^{\mathbf{c}} G_{\epsilon, s} \rightarrow \mathcal{C}_{0}^{\mathbf{c}} \mathcal{B}^{2}$ gives rise to a functor $\mathcal{C}^{\mathbf{c}} G_{\epsilon, s} \rightarrow \mathcal{C}^{\mathbf{c}} \mathcal{B}^{2}$ denoted again by $\underline{\zeta}_{\epsilon, s}$ (it is again called truncated restriction).

The operation $K * K^{\prime}$ is again called truncated convolution. It has a canonical associativity isomorphism (deduced from that in Proposition 3.7) which satisfies the pentagon property.

The operation $L \bullet L^{\prime}$ makes $\mathcal{C}^{\mathbf{c}} \mathcal{B}^{2}$ into a monoidal abelian category (see also [L9]) which has a unit object (see [L13, 9.2]) and is rigid (see [L13, 9.3]).

Note that $L \mapsto{ }^{\epsilon} L$ (see 1.6) can be regarded as a functor $\mathcal{C}^{\mathbf{c}} \mathcal{B}^{2} \rightarrow \mathcal{C}^{\mathbf{c}} \mathcal{B}^{2}$.

6.2. Extending slightly a definition in [Mu, 3.1] we define a $\epsilon$-half braiding for an object $\mathcal{L} \in \mathcal{C}^{\mathbf{c}} \mathcal{B}^{2}$ as a collection $e_{\mathcal{L}}=\left\{e_{\mathcal{L}}(L) ; L \in \mathcal{C}^{\mathbf{c}} \mathcal{B}^{2}\right\}$ where $e_{\mathcal{L}}(L)$ are isomorphisms ${ }^{\epsilon} L \bullet \mathcal{L} \stackrel{\sim}{\longrightarrow} \mathcal{L} \bullet L$ such that (i) and (ii) below hold:

(i) If $L \stackrel{t}{\longrightarrow} L^{\prime}$ is any morphism in $\mathcal{C}^{\mathbf{c}} \mathcal{B}^{2}$, then the diagram

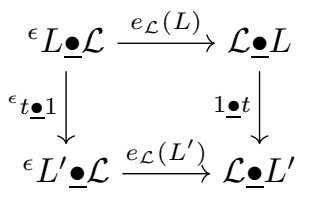

is commutative.

(ii) If $L, L^{\prime} \in \mathcal{C}^{\mathbf{c}} \mathcal{B}^{2}$, then $e_{\mathcal{L}}\left(L \bullet L^{\prime}\right):{ }^{\epsilon}\left(L \bullet L^{\prime}\right) \bullet \mathcal{L} \rightarrow \mathcal{L} \underline{\bullet}\left(L \bullet L^{\prime}\right)$ is equal to the composition

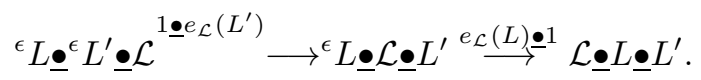

When $\epsilon=1$, this reduces to the definition of a half-braiding for $\mathcal{L}$ given in [Mu, 3.1]. Let $\mathcal{Z}_{\epsilon}^{\mathbf{c}}$ the category whose objects are the pairs consisting of an object $\mathcal{L}$ of $\mathcal{C}^{\mathbf{c}} \mathcal{B}^{2}$ and an $\epsilon$-half braiding for $\mathcal{L}$. For $\left(\mathcal{L}, e_{\mathcal{L}}\right),\left(\mathcal{L}^{\prime}, e_{\mathcal{L}^{\prime}}\right)$ in $\mathcal{Z}_{\epsilon}^{\mathbf{c}}$ we define 
$\operatorname{Hom}_{\mathcal{Z}_{\epsilon}^{\mathrm{c}}}\left(\left(\mathcal{L}, e_{\mathcal{L}}\right),\left(\mathcal{L}^{\prime}, e_{\mathcal{L}^{\prime}}\right)\right)$ to be the vector space consisting of all $t \in \operatorname{Hom}_{\mathcal{C}^{\mathrm{c}} \mathcal{B}^{2}}\left(\mathcal{L}, \mathcal{L}^{\prime}\right)$ such that for any $L \in \mathcal{C}^{\mathbf{c}} \mathcal{B}^{2}$ the diagram

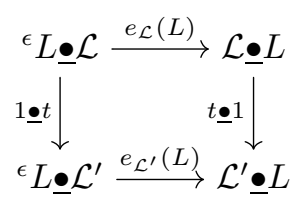

is commutative. We say that $\mathcal{Z}_{\epsilon}^{\mathbf{c}}$ is the $\epsilon$-centre of $\mathcal{C}^{\mathbf{c}} \mathcal{B}^{2}$. (When $\epsilon=1$, it reduces to the centre of $\mathcal{C}^{\mathbf{c}} \mathcal{B}^{2}$; see $[\mathrm{Mu}, 3.2]$.)

If $s \in \mathbf{Z}$ and $K \in \mathcal{C}^{\mathbf{c}} G_{s}$, then the isomorphisms Proposition 2.9(a) provide an $\epsilon$-half braiding for $\underline{\zeta}_{\epsilon, s}(K) \in \mathcal{C}^{\mathbf{c}} \mathcal{B}^{2}$ so that $\underline{\zeta}_{\epsilon, s}(K)$ can be naturally viewed as an object of $\mathcal{Z}_{\epsilon}^{\mathrm{c}}$ denoted by $\overline{\underline{\zeta}_{\epsilon, s}(K)}$. (Note that 2.9 is stated in the mixed category but, as above, it implies the corresponding result in the unmixed category.) Then $K \mapsto \overline{\zeta_{\epsilon, s}(K)}$ is a functor $\mathcal{C}^{\mathbf{c}} G_{\epsilon, s} \rightarrow \mathcal{Z}_{\epsilon}^{\mathbf{c}}$. We have the following result.

Theorem 6.3. Let $s \in \mathbf{Z}$. The functor $\mathcal{C}^{\mathbf{c}} G_{\epsilon, s} \rightarrow \mathcal{Z}_{\epsilon}^{\mathbf{c}}, K \mapsto \overline{\zeta_{\epsilon, s}(K)}$ is an equivalence of abelian categories.

When $\epsilon=1, s=0$ this reduces to [L13, 9.5]. The general case will be proved in 6.5 .

Note that, when combined with 1.6(b), the theorem yields for any $F \in G_{\epsilon, s}$ (with $s>0$ ) an equivalence of abelian categories

$$
\operatorname{Rep}^{\mathbf{c}}\left(G^{F}\right) \stackrel{\sim}{\longrightarrow} \mathcal{Z}_{\epsilon}^{\mathbf{c}}
$$

6.4. By a variation of a general result on semisimple rigid monoidal categories in ENO, Proposition 5.4], for any $L \in \mathcal{C}^{\mathbf{c}} \mathcal{B}^{2}$ one can define directly an $\epsilon$-half braiding on the object $I_{\epsilon}(L):=\bigoplus_{y \in \mathbf{c}} \mathbf{L}_{y} \bullet L \bullet \mathbf{L}_{\epsilon(y)^{-1}}$ of $\mathcal{C}^{\mathbf{c}} \mathcal{B}^{2}$ such that, denoting by $\overline{I_{\epsilon}(L)}$ the corresponding object of $\mathcal{Z}_{\epsilon}^{\mathbf{c}}$, we have canonically

$$
\operatorname{Hom}_{\mathcal{C}^{\mathrm{c}} \mathcal{B}^{2}}(L, \mathcal{L})=\operatorname{Hom}_{\mathcal{Z}_{\epsilon}^{\mathrm{c}}}\left(\overline{I_{\epsilon}(L)},\left(\mathcal{L}, e_{\mathcal{L}}\right)\right)
$$

for any $\left(\mathcal{L}, e_{\mathcal{L}}\right) \in \mathcal{Z}_{\epsilon}^{\mathbf{c}}$.

The $\epsilon$-half braiding on $I_{\epsilon}(L)$ can be described as follows: for any $L^{\prime} \in \mathcal{C}^{\mathbf{c}} \mathcal{B}^{2}$ we have canonically

$$
\begin{aligned}
& { }^{\epsilon} L^{\prime} \bullet I_{\epsilon}(L)=\bigoplus_{y \in \mathbf{c}}\left({ }^{\epsilon} L^{\prime} \bullet \mathbf{L}_{y} \bullet L \bullet \mathbf{L}_{\epsilon(y)^{-1}}\right) \\
& =\bigoplus_{y, z \in \mathbf{c}} \operatorname{Hom}_{\mathcal{C}^{\mathbf{c}} \mathcal{B}^{2}}\left(\mathbf{L}_{z},{ }^{\epsilon} L^{\prime} \bullet \mathbf{L}_{y}\right) \otimes\left(\mathbf{L}_{z} \bullet L \underline{\bullet}_{\epsilon(y)^{-1}}\right) \\
& =\bigoplus_{y, z \in \mathbf{c}} \operatorname{Hom}_{\mathcal{C}^{\mathbf{c}} \mathcal{B}^{2}}\left(\mathbf{L}_{y^{-1}}, \mathbf{L}_{z^{-1}} \bullet^{\epsilon} L^{\prime}\right) \otimes\left(\mathbf{L}_{z} \bullet L \bullet \mathbf{L}_{\epsilon(y)^{-1}}\right) \\
& =\bigoplus_{y, z \in \mathbf{c}} \operatorname{Hom}_{\mathcal{C}^{\mathbf{c}} \mathcal{B}^{2}}\left(\mathbf{L}_{\epsilon(y)^{-1}}, \mathbf{L}_{\epsilon(z)^{-1}} \bullet L^{\prime}\right) \otimes\left(\mathbf{L}_{z} \bullet L \bullet \mathbf{L}_{\epsilon(y)^{-1}}\right) \\
& =\bigoplus_{z \in \mathbf{c}}\left(\mathbf{L}_{z} \bullet L \bullet \mathbf{L}_{\epsilon(z)^{-1}} \bullet L^{\prime}\right)=I_{\epsilon}(L) \bullet L^{\prime} .
\end{aligned}
$$

(We have used [L13, 7.7].) By a variation of results in [Mu, 3.3], ENO, 2.15], we see that $\mathcal{Z}_{\epsilon}^{\mathbf{c}}$ is a semisimple $\overline{\mathbf{Q}}_{l}$-linear category with finitely many simple objects up to isomorphism. Note that 
(b) if $\sigma=\left(\mathcal{L}, e_{\mathcal{L}}\right)$ is a simple object of $\mathcal{Z}_{\epsilon}^{\mathbf{c}}$, then $\sigma$ is a summand of $\overline{I_{\epsilon}\left(\mathbf{L}_{z}\right)}$ for some $z \in \mathbf{c}$.

Indeed, let $z \in \mathbf{c}$ be such that $\mathbf{L}_{z}$ is a summand of $\mathcal{L}$ in $\mathcal{C}^{\mathbf{c}} \mathcal{B}^{2}$; then by (a), $\sigma$ is a summand of $\overline{I_{\epsilon}\left(\mathbf{L}_{z}\right)}$.

6.5. Let $s \in \mathbf{Z}$. For $x \in \mathbf{c}$ we have canonically $\underline{\zeta}_{\epsilon, s} \underline{\chi}_{\epsilon, s} \mathbf{L}_{x}=I_{\epsilon}\left(\mathbf{L}_{x}\right)$ as objects of $\mathcal{C}^{\mathbf{c}} \mathcal{B}^{2}$; see Theorem 4.8. This identification is compatible with the $\epsilon$-half braidings (see $6.2,6.4$ ). (When $\epsilon=1, s=0$ this follows from the last commutative diagram in [L13, 7.9]; in the general case we have an analogous commutative diagram, which is established using the results in Section 4.) It follows that

(a)

$$
\overline{\bar{\zeta}_{\epsilon, s} \underline{\chi}_{\epsilon, s} \mathbf{L}_{x}}=\overline{I_{\epsilon}\left(\mathbf{L}_{x}\right)} \text {. }
$$

Using this and $6.4\left(\right.$ a) with $\mathcal{L}=\overline{\zeta_{\epsilon, s} \underline{\chi}_{\epsilon, s} \tilde{L}}, \tilde{L} \in \mathcal{C}^{\mathbf{c}} \mathcal{B}^{2}$, we see that

$$
\operatorname{Hom}_{\mathcal{C}^{\mathbf{c}} \mathcal{B}^{2}}\left(\mathbf{L}_{x}, \underline{\zeta}_{\epsilon, s} \underline{\chi}_{\epsilon, s} \tilde{L}\right)=\operatorname{Hom}_{\mathcal{Z}_{\epsilon}^{\mathrm{c}}}\left(\overline{\zeta_{\epsilon, s} \underline{\chi}_{\epsilon, s} \mathbf{L}_{x}}, \overline{\zeta_{\epsilon, s} \underline{\chi}_{\epsilon, s} \tilde{L}}\right) \text {. }
$$

Combining this with Proposition 5.3 we obtain for $\tilde{L}=\mathbf{L}_{x^{\prime}}\left(\right.$ with $\left.x^{\prime} \in \mathbf{c}\right)$ :

$$
{\stackrel{\circ}{A}, x^{\prime}}=\stackrel{\circ}{A}_{x, x^{\prime}}^{\prime}
$$

where

$$
\stackrel{\circ}{A}_{x, x^{\prime}}=\operatorname{Hom}_{\mathcal{C}^{\mathrm{c}} G_{\epsilon, s}}\left(\underline{\chi}_{\epsilon, s}\left(\mathbf{L}_{x}\right), \underline{\chi}_{\epsilon, s}\left(\mathbf{L}_{x^{\prime}}\right)\right), \stackrel{\circ}{A}_{x, x^{\prime}}^{\prime}=\operatorname{Hom}_{\mathcal{Z}_{\epsilon}^{\mathrm{c}}}\left(\overline{\underline{\zeta}_{\epsilon, s} \underline{\chi}_{\epsilon, s} \mathbf{L}_{x}}, \overline{\zeta_{\epsilon, s} \underline{\chi}_{\epsilon, s} \mathbf{L}_{x^{\prime}}}\right) .
$$

Note that the identification (b) is induced by the functor $K \mapsto \overline{\zeta_{\epsilon, s}(K)}$. Let $\stackrel{\AA}{A}=$ $\bigoplus_{x, x^{\prime} \in \mathbf{c}} \stackrel{\circ}{A}_{x, x^{\prime}}, \stackrel{\circ}{A}^{\prime}=\bigoplus_{x, x^{\prime} \in \mathbf{c}} \stackrel{\circ}{A}_{x, x^{\prime}}$. Then from (b) we have $\stackrel{\circ}{A}=\stackrel{\circ}{A}^{\prime}$. Note that this identification is compatible with the obvious algebra structures of $\AA$. $\AA^{\prime}$.

For any $A \in C S_{\epsilon, s, \mathbf{c}}$ we denote by $\stackrel{\circ}{A}_{A}$ the set of all $f \in \stackrel{\circ}{A}$ such that for any $x, x^{\prime}$, the $\left(x, x^{\prime}\right)$-component of $f$ maps the $A$-isotypic component of $\underline{\chi}_{\epsilon, s}\left(\mathbf{L}_{x}\right)$ to the $A$-isotypic component of $\underline{\chi}_{\epsilon, s}\left(\mathbf{L}_{x^{\prime}}\right)$ and any other isotypic component of $\underline{\chi}_{\epsilon, s}\left(\mathbf{L}_{x}\right)$ to 0 . Then $\stackrel{\circ}{A}=\bigoplus_{A \in C S_{\epsilon, s, \mathbf{c}}} \stackrel{\circ}{A}_{A}$ is the decomposition of $\stackrel{\circ}{A}$ into a sum of simple algebras (each $\stackrel{\circ}{A}_{A}$ is $\neq 0$ since, by 1.5 (b) and $1.8(\mathrm{a})$, any $A$ is a summand of some $\left.\underline{\chi}_{\epsilon, s}\left(\mathbf{L}_{x}\right)\right)$.

Let $\mathfrak{S}$ be a set of representatives for the isomorphism classes of simple objects of $\mathcal{Z}_{\epsilon}^{\mathbf{c}}$. For any $\sigma \in \mathfrak{S}$ we denote by $\AA_{\sigma}^{\prime}$ the set of all $f^{\prime} \in \stackrel{\circ}{A}^{\prime}$ such that for any $x, x^{\prime}$, the $\left(x, x^{\prime}\right)$-component of $f^{\prime}$ maps the $\sigma$-isotypic component of $\overline{\underline{\zeta}_{\epsilon, s} \underline{\chi}_{\epsilon, s}\left(\mathbf{L}_{x}\right)}$ to the $\sigma$-isotypic component of $\overline{\zeta_{\epsilon, s} \underline{\chi}_{\epsilon, s}\left(\mathbf{L}_{x^{\prime}}\right)}$ and any other isotypic component of $\overline{\zeta_{\epsilon, s} \underline{\chi}_{\epsilon, s}\left(\mathbf{L}_{x}\right)}$ to 0 . Then $\stackrel{\circ}{A}^{\prime}=\bigoplus_{\sigma \in \mathfrak{S}} \stackrel{\circ}{A}_{\sigma}^{\prime}$ is the decomposition of $\stackrel{\circ}{A}^{\prime}$ into a sum of simple algebras (each $\stackrel{\circ}{\sigma}_{\sigma}^{\prime}$ is $\neq 0$ since any $\sigma$ is a summand of some $\overline{\underline{\zeta}_{\epsilon, s} \underline{\chi}_{\epsilon, s}\left(\mathbf{L}_{z}\right)}$ (we use $6.4(\mathrm{~b}), 6.5(\mathrm{a}))$.

Since $\AA=\AA^{\prime}$, from the uniqueness of decomposition of a semisimple algebra as a direct sum of simple algebras, we see that there is a unique bijection $C S_{\epsilon, s, \mathbf{c}} \leftrightarrow \mathfrak{S}$, $A \leftrightarrow \sigma_{A}$ such that the identification $\stackrel{\circ}{A}=\stackrel{\circ}{A}^{\prime}$ restricts to an identification $\AA_{A}=\stackrel{\circ}{A}_{\sigma_{A}}^{\prime}$ for any $A \in C S_{\epsilon, s, \mathbf{c}}$. From the definitions we now see that for any $A \in C S_{\epsilon, s, \mathbf{c}}$ we have $\overline{\zeta_{\epsilon, s} A} \cong \sigma_{A}$. Therefore Theorem 6.3 holds.

Theorem 6.6. Let $s \in \mathbf{Z}$. Let $L \in \mathcal{C}^{\mathbf{c}} \mathcal{B}^{2}, K \in \mathcal{C}^{\mathbf{c}} G_{\epsilon, s}$. We have canonically

$$
\operatorname{Hom}_{\mathcal{C}^{\mathbf{c}} \mathcal{B}^{2}}\left(L, \underline{\zeta}_{\epsilon, s}(K)\right)=\operatorname{Hom}_{\mathcal{C}^{\mathbf{c}} G_{\epsilon, s}}\left(\underline{\chi}_{\epsilon, s}(L), K\right) \text {. }
$$


Moreover, in $\mathcal{C}^{\mathbf{c}} \mathcal{B}^{2}$ we have $\underline{\zeta}_{e, s}(K) \cong \bigoplus_{z \in \mathbf{c}^{0}} \mathbf{L}_{z}^{\oplus m_{z}}$ where $\mathbf{c}^{0}$ is as in 1.5 and $m_{z} \in \mathbf{N}$.

From Theorems 6.3 and 6.5 , we see that

$$
\begin{aligned}
& \operatorname{Hom}_{\mathcal{C}^{\mathrm{c}} G_{\epsilon, s}}\left(\underline{\chi}_{\epsilon, s}(L), K\right)=\operatorname{Hom}_{\mathcal{Z}_{\epsilon}^{\mathrm{c}}}\left(\overline{\underline{\zeta}_{\epsilon, s} \underline{\chi}_{\epsilon, s}(L)}, \overline{\underline{\zeta}_{\epsilon, s} K}\right) \\
& =\operatorname{Hom}_{\mathcal{Z}_{\epsilon}^{\mathrm{c}}}\left(\overline{I_{\epsilon}(L)}, \overline{\underline{\zeta}_{\epsilon, s} K}\right) .
\end{aligned}
$$

Using 6.4(a) we see that

$$
\operatorname{Hom}_{\mathcal{Z}_{\epsilon}^{\mathrm{c}}}\left(\overline{I_{\epsilon}(L)}, \overline{\underline{\zeta}_{\epsilon, s} K}\right)=\operatorname{Hom}_{\mathcal{C}^{\mathrm{c}} \mathcal{B}^{2}}\left(L, \underline{\zeta}_{\epsilon, s}(K)\right)
$$

and (a) follows. To prove the second assertion of the theorem it is enough to show that for any $z \in \mathbf{c}-\mathbf{c}^{0}$ we have $\operatorname{Hom}_{\mathcal{C}^{c} \mathcal{B}^{2}}\left(\mathbf{L}_{z}, \underline{\zeta}_{\epsilon, s}(K)\right)=0$; by (a), it is enough to show that $\underline{\chi}_{\epsilon, s}\left(\mathbf{L}_{z}\right)=0$ and this follows from 1.5(c). (The case where $\epsilon=1, s=0$ is just [L13, 9.8].)

6.7. Let $s \in \mathbf{Z}$. For $K \in \mathcal{C}^{\mathbf{c}} G_{\epsilon, s}$ we have canonically

$$
\mathfrak{D}\left(\underline{\zeta}_{\epsilon, s}(\mathfrak{D}(K))\right)=\underline{\zeta}_{\epsilon, s}(K) .
$$

When $\epsilon=1, s=0$ this is proved in [L13, 9.9]. The proof in the general case is entirely similar; it uses Theorem 6.6(a) and 1.10(a).

6.8. In this subsection we assume that $\epsilon=1$. The monoidal structure on $\mathcal{C}^{\mathbf{c}} \mathcal{B}^{2}$ induces a monoidal structure on $\mathcal{Z}_{1}^{\mathrm{c}}$. Moreover, the category

$$
\bigsqcup_{s \in \mathbf{Z}} \mathcal{C}^{\mathbf{c}} G_{1, s}=\mathcal{C}^{\mathbf{c}} G_{1,0} \bigsqcup_{s \in \mathbf{Z} ; s \neq 0} \operatorname{Rep}^{\mathbf{c}}\left(G^{F_{0}^{s}}\right)
$$

(see 1.6(b)) has a monoidal structure given by truncated convolution; see 6.1. Moreover, Theorem 6.3 provides a functor from (a) to $\mathcal{Z}_{1}^{\mathbf{c}}$ which is an equivalence when restricted to any $\mathcal{C}^{\mathbf{c}} G_{s}$. This functor is compatible with the monoidal structures (this can be deduced from Proposition 3.8 and from the fact that the monoidal structure of $\mathcal{Z}_{1}^{\mathbf{c}}$ is equivalent to its opposite). Note that $\mathcal{C}^{\mathbf{c}} G_{1,0}$ is a monoidal subcategory of (a), whose unit object, described in [L13, 9.10], is also a unit object for the monoidal category (a).

6.9. The functor $L \mapsto{ }^{\epsilon} L$ from $\mathcal{C}^{\mathbf{c}} \mathcal{B}^{2}$ into itself induces a functor $\mathcal{Z}_{\epsilon}^{\mathbf{c}} \rightarrow \mathcal{Z}_{\epsilon}^{\mathbf{c}}$ which carries any simple object $\left(L, e_{L}\right)$ of $\mathcal{Z}_{\epsilon}^{\mathbf{c}}$ into an object isomorphic to $\left(L, e_{L}\right)$; this follows from 2.10(c), using Theorem 6.3.

6.10. Let $s \in \mathbf{Z}$. For any $A \in C S_{\epsilon, s, \mathbf{c}}$ and any $x \in \mathbf{c}$ we denote by $n_{A, x}$ the multiplicity of $A$ in $\chi_{\epsilon, s} \mathbf{L}_{x} \in \mathcal{C}^{\mathbf{c}} G_{\epsilon, s}$. From Theorem 6.3 and its proof we see that if $\sigma$ is the simple object of $\mathcal{Z}_{\epsilon}^{\mathbf{c}}$ corresponding to $A$, then $n_{A, x}$ is equal to the multiplicity of $\sigma$ in $\overline{I_{\epsilon}\left(\mathbf{L}_{x}\right)} \in \mathcal{Z}_{\epsilon}^{\mathrm{c}}$.

\section{Relation with Soergel Bimodules}

7.1. Let $R$ be the algebra of polynomials functions on a fixed reflection representation of $W$ (over $\overline{\mathbf{Q}}_{l}$ ). Then for each $x \in W$, the indecomposable Soergel graded $R$-bimodule $B_{x}$ is defined as in [So, 6.16]. Let $C_{\mathbf{c}}$ be the category of graded $R$ bimodules which are isomorphic to finite direct sums of graded $R$-bimodules of the form $B_{x}(x \in \mathbf{c})$ without shift. There is a well defined functor $M \mapsto{ }^{\epsilon} M$ from $C_{\mathbf{c}}$ to $C_{\mathbf{c}}$ which is linear and satisfies ${ }^{\epsilon} B_{x}=B_{\epsilon^{-1}(x)}$ for $x \in \mathbf{c}$. Now $C_{\mathbf{c}}$ has a 
natural monoidal structure (see [L13, 10.1]) defined purely in terms of $R, W$, c. (Its definition makes use of the results in [EW].) From the definition we see that $C_{\mathbf{c}}$ is equivalent to $\mathcal{C}^{\mathbf{c}} \mathcal{B}^{2}$ as monoidal categories so that $M \mapsto{ }^{\epsilon} M$ corresponds to $L \mapsto{ }^{\epsilon} L$ from $\mathcal{C}^{\mathbf{c}} \mathcal{B}^{2}$ to itself. Then the $\epsilon$-centre of $C_{\mathbf{c}}$ is defined as in 6.2. It is naturally equivalent to $\mathcal{Z}_{\epsilon}^{\mathbf{c}}$. Thus we can restate Theorem 6.3 as follows.

(a) For any $s \in \mathbf{Z}$, the category $\mathcal{C}^{\mathbf{c}} G_{\epsilon, s} \rightarrow \mathcal{Z}_{\epsilon}^{\mathbf{c}}$ is naturally equivalent to the $\epsilon$-centre of the monoidal category $C_{\mathbf{c}}$.

This, combined with 1.6(b), shows that for $F \in G_{\epsilon, s}$ (with $s>0$ ), the category $\operatorname{Rep}^{\mathbf{c}}\left(G^{F}\right)$ is equivalent to the $\epsilon$-centre of the monoidal category $C_{\mathbf{c}}$; thus, the set of simple objects of $\operatorname{Rep}^{\mathbf{c}}\left(G^{F}\right)$ is not only independent of $s$ but also independent of the characteristic of $\mathbf{k}$, since the $\epsilon$-centre of $C_{\mathbf{c}}$ is so. (Here we identify $\overline{\mathbf{Q}}_{l}$ with the complex numbers.)

7.2. As mentioned in [L13, 10.1], the definition of the monoidal category $C_{\mathbf{c}}$ makes sense even when $W$ is replaced by any (say finite, irreducible) Coxeter group and c is a two-sided cell in $W$. Assume now that $\epsilon: W \rightarrow W$ is an automorphism of $W$ which leaves stable the set of simple reflections and leaves stable c. Then the definition of the $\epsilon$-centre of $C_{\mathbf{c}}$ makes sense even if $W$ is noncrystallographic. We expect that the indecomposable objects of the $\epsilon$-centre of $C_{\mathbf{c}}$ are in bijection with the "unipotent characters" associated to $W, \epsilon, \mathbf{c}$ in [L5]. (For $\epsilon=1$ this expectation has already been stated in [L13, 10.1].)

\section{REFERENCES}

[BBD] A. A. Beilinson, J. Bernstein, and P. Deligne, Faisceaux pervers (French), Analysis and topology on singular spaces, I (Luminy, 1981), Astérisque, vol. 100, Soc. Math. France, Paris, 1982, pp. 5-171. MR751966 (86g:32015)

[BFO] R. Bezrukavnikov, M. Finkelberg, and V. Ostrik, Character D-modules via Drinfeld center of Harish-Chandra bimodules, Invent. Math. 188 (2012), no. 3, 589-620, DOI 10.1007/s00222-011-0354-3. MR2917178

[DL] P. Deligne and G. Lusztig, Representations of reductive groups over finite fields, Ann. of Math. (2) 103 (1976), no. 1, 103-161. MR0393266 (52 \#14076)

[EW] B. Elias and G. Williamson, The Hodge theory of Soergel bimodules, Ann. of Math. (2) 180 (2014), no. 3, 1089-1136, DOI 10.4007/annals.2014.180.3.6. MR.3245013

[ENO] P. Etingof, D. Nikshych, and V. Ostrik, On fusion categories, Ann. of Math. (2) 162 (2005), no. 2, 581-642, DOI 10.4007/annals.2005.162.581. MR2183279 (2006m:16051)

[L1] G. Lusztig, Characters of reductive groups over a finite field, Annals of Mathematics Studies, vol. 107, Princeton University Press, Princeton, NJ, 1984. MR742472 (86j:20038)

[L2] G. Lusztig, Character sheaves. II, Adv. in Math. 57 (1985), no. 3, 226-265, DOI 10.1016/0001-8708(85)90064-7. MR806210(87m:20118a)

[L3] G. Lusztig, Character sheaves. III, Adv. in Math. 57 (1985), no. 3, 266-315, DOI 10.1016/0001-8708(85)90064-7. MR806210 (87m:20118a)

[L4] G. Lusztig, Character sheaves. IV, Adv. in Math. 59 (1986), no. 1, 1-63, DOI 10.1016/0001-8708(86)90036-8. MR825086 (87m:20118b)

[L5] George Lusztig, Coxeter groups and unipotent representations, Astérisque 212 (1993), 191-203.

[L6] G. Lusztig, Character sheaves on disconnected groups. VII, Represent. Theory 9 (2005), 209-266, DOI 10.1090/S1088-4165-05-00278-5. MR2133758(2006e:20089)

[L7] G. Lusztig, Character sheaves on disconnected groups. IX, Represent. Theory 10 (2006), 353-379, DOI 10.1090/S1088-4165-06-00315-3. MR2240705 (2008e:20078)

[L8] G. Lusztig, Character sheaves on disconnected groups. X, Represent. Theory 13 (2009), 82-140, DOI 10.1090/S1088-4165-09-00348-3. MR2495562 (2010i:20058)

[L9] G. Lusztig, Cells in affine Weyl groups and tensor categories, Adv. Math. 129 (1997), no. 1, 85-98, DOI 10.1006/aima.1997.1645. MR.1458414 (98f:20030) 
[L10] G. Lusztig, Hecke algebras with unequal parameters, CRM Monograph Series, vol. 18, American Mathematical Society, Providence, RI, 2003. MR1974442 (2004k:20011)

[L11] G. Lusztig, On certain varieties attached to a Weyl group element, Bull. Inst. Math. Acad. Sin. (N.S.) 6 (2011), no. 4, 377-414. MR2907958

[L12] G. Lusztig, Restriction of a character sheaf to conjugacy classes, Bull. Soc. Math. Roum., Tome 58 (2015), no. 3, pp. 297-309.

[L13] G. Lusztig, Truncated convolution of character sheaves, Bull. Inst. Math. Acad. Sin. (N.S.) 10 (2015), no. 1, 1-72. MR3309291

[L14] G. Lusztig, Non-unipotent character sheaves as a categorical centre, arxiv: 1506.04598.

[Mu] M. Müger, From subfactors to categories and topology. II. The quantum double of tensor categories and subfactors, J. Pure Appl. Algebra 180 (2003), no. 1-2, 159-219, DOI 10.1016/S0022-4049(02)00248-7. MR.1966525 (2004f:18014)

[So] W. Soergel, Kazhdan-Lusztig-Polynome und unzerlegbare Bimoduln über Polynomringen (German, with English and German summaries), J. Inst. Math. Jussieu 6 (2007), no. 3, 501-525, DOI 10.1017/S1474748007000023. MR2329762(2009c:20009)

Department of Mathematics, M.I.T., Cambridge, Massachusetts 02139

E-mail address: gyuri@math.mit.edu 